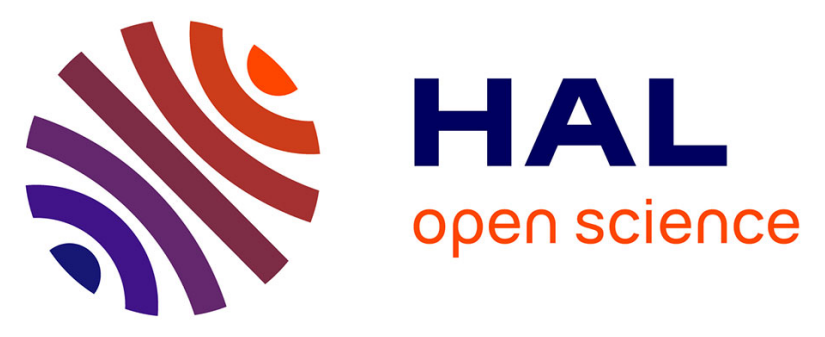

\title{
Predicted airway obstruction distribution based on dynamical lung ventilation data: a coupled modeling-machine learning methodology
}

Nicolas Pozin, Spyridon Montesantos, Ira Katz, Marine Pichelin, Irene

Vignon-Clementel, Céline Grandmont

\section{To cite this version:}

Nicolas Pozin, Spyridon Montesantos, Ira Katz, Marine Pichelin, Irene Vignon-Clementel, et al.. Predicted airway obstruction distribution based on dynamical lung ventilation data: a coupled modelingmachine learning methodology. International Journal for Numerical Methods in Biomedical Engineering, 2018, 34 (9), 10.1002/cnm.3108 . hal-01568065v3

\section{HAL Id: hal-01568065 \\ https://hal.science/hal-01568065v3}

Submitted on 25 May 2018

HAL is a multi-disciplinary open access archive for the deposit and dissemination of scientific research documents, whether they are published or not. The documents may come from teaching and research institutions in France or abroad, or from public or private research centers.
L'archive ouverte pluridisciplinaire HAL, est destinée au dépôt et à la diffusion de documents scientifiques de niveau recherche, publiés ou non, émanant des établissements d'enseignement et de recherche français ou étrangers, des laboratoires publics ou privés. 


\title{
Predicted airway obstruction distribution based on dynamical lung ventilation data: a coupled modeling-machine learning methodology
}

\author{
N. Pozin ${ }^{1,2,3}$, S. Montesantos ${ }^{3}$, I. Katz ${ }^{3,4}$, M. Pichelin ${ }^{3}$, I. Vignon-Clementel ${ }^{1,2}$, C. Grandmont ${ }^{1,2}$ \\ ${ }^{1}$ INRIA Paris, 2 Rue Simone Iff, 75012 Paris, France \\ ${ }^{2}$ Sorbonne Universités, UPMC Univ. Paris 6, Laboratoire Jacques-Louis Lions, 75252 Paris, France \\ ${ }^{3}$ Medical R\&D, WBL Healthcare, Air Liquide Santé International, 1 Chemin de la Porte des Loges, 78350 Les Loges-en- \\ Josas, France \\ ${ }^{4}$ Department of Mechanical Engineering, Lafayette College, Easton, PA, 18042, USA
}

\begin{abstract}
In asthma and COPD, some airways of the tracheo-bronchial tree can be constricted, from moderate narrowing up to closure. Those pathological patterns of obstructions affect the lung ventilation distribution. While some imaging techniques enable visualization and quantification of constrictions in proximal generations, no non-invasive technique exists to provide the airway morphology and obstruction distribution in distal areas. In this work, we propose a method that exploits lung ventilation measures to access positions of airways obstructions (restrictions and closures) in the tree. This identification approach combines a lung ventilation model, in which a $0 D$ tree is strongly coupled to a $3 D$ parenchyma description, along with a machine learning approach. Based on synthetic data generated with typical temporal and spatial resolutions as well as reconstruction errors, we obtain very encouraging results of the obstruction distribution, with a detection rate higher than $85 \%$.
\end{abstract}

\section{Introduction}

Pathologies such as asthma or COPD can induce airway constriction [1], [2] that generate ventilation defects [3], [4]. However, in clinical practice the airway obstruction distribution is largely unknown. As the response to Heliox treatment may depend on airway closure severity and position within the lung (generational and regional position) [5]; knowing the airway obstruction distribution could give predictive insights on the treatment efficiency. Another clinical application of the airway obstruction distribution could be to predict aerosol deposition, which can be greatly affected by bronchoconstrictions [6]. High resolution computed tomography (HRCT) can show the presence of constrictions in asthmatic patients [7], [8] but only proximal airways are easily accessible with current resolutions. However, given the fact that histological analysis demonstrates that more distal branches can be constricted [9], [10], greater resolution is needed. There does exist dynamic imaging techniques such as $4 D$ computed tomography (4D-CT) [11], [12] and $4 D$ magnetic resonance imaging (4D-MRI) [13], [14] that can provide information on lung ventilation distribution during the breathing cycle.

In this work, we propose an approach that exploits $4 D$ dynamical ventilation data to identify which regions of the tracheo-bronchial tree are constricted or plugged; that is, the distribution of obstructions in the airways. The process combines a lung ventilation model presented in [15], in which a $0 D$ model of the tracheo-bronchial tree is coupled to a $3 D$ continuous elastic media representing the lung parenchyma, along with a plug identification step that includes a supervised machine learning technique. Approaches combining machine learning and modeling have been proposed to speed up calculations [16] or to improve 
diagnosis quality [17]. To the best of our knowledge, this study is the first attempt to use lung ventilation data in order to develop insight into the tree structure.

In Section 2, the global methodology is presented. In Section 3 the ventilation model and the theoretical background of the plug identification approach are described. Section 4 gives results on synthetic data.

\section{Direct ventilation model and identification problem approaches}

\subsection{Global methodology}

The tracheo-bronchial tree is a dyadic structure starting from large upper airways, referred to as proximal, down to smaller branches, referred to as distal. Some pathologies affect the tree structure; in asthma for instance, airways can be constricted. This goes from frequent moderate constrictions throughout the tree with airway radii reductions of 10 to $25 \%$ on average [18], [2] up to, in a low proportion of cases, complete closure [10], [8]. These latter severe constrictions will be designated as plugs. As observed experimentally through dynamic $4 D$ images [11], [13], [14], obstructions affect the lung ventilation distribution. It takes more energy for the gas to flow through a constricted path, and thus downstream parenchyma regions are less irrigated.

Simulation results presented in subsection 4.1 indicate that ventilation defects caused by constrictions are mainly due to plugs rather than moderate constrictions, although the former are less frequent. Thus, ventilation information provided by $4 D$ scans may give insights on the plug distribution. While the ventilation model described in subsection 2.2 provides the flow distribution knowing the constriction repartition within the tree, we aim at using the ventilation data (as those provided by medical images for instance) in order to determine which airways are significantly constricted. This is an identification problem for which unknowns are the tree branch radii (see subsection 2.3). There is inter-variability in airway dimensions [19] and typical normal radii values are within a prescribed range. Here the healthy variability is neglected (see Remark 4) and each airway in the healthy state is assumed to have a known representative value. The difference between the pathological radius and the healthy one then reveals that constriction exists.

Radii are accessed through the determination of airway resistances. As to be explained in subsection 2.2, flows at the tree exits and pressure drops from the tree entrance to the exits are linked through airway resistances by:

$$
A Q=P
$$

where $Q=\left(q_{i}\right)_{i}$ with $q_{i}$ the flow at the $i^{t h}$ exit. The $i^{t h}$ element of $P$ is the pressure drop from the tree entrance to the $i^{\text {th }}$ exit. Matrix $A$ is defined by (6) and includes information on the tree resistance distribution. Knowing $Q$ and $P$, one can get insights on airway resistances and hence on the constrictions through solution of an identification problem. The flux $Q$ can be computed from imaging data. Indeed, from $4 D$ scans, registration techniques can provide the parenchyma displacement field and finally $Q$ through equation (5) (see subsection 2.2). Regarding the pressure $P$, in this study, we propose to determine it through a minimization problem (see subsection 2.3) based on the ventilation model described in [15] and subsection 2.2. In subsection 2.3 , it is shown that for the resistance identification problem to be well-posed the maximum number of unknowns is, at least, two times smaller than the number of airways within the tree. Thus only a sub-set of the branches can have an unknown radius. To select them, an a priori prediction process is proposed. Based on ventilation features extracted from $4 D$ scans (see subsection 2.4.1), a machine learning technique determines which airways are more likely to be constricted (see 
subsection 2.4.2). If the prediction process is good, non-selected airways are minimally constricted. Since their radii cannot be set among the unknowns, they are assumed to have known dimensions that are set in the normal physiological range. The a priori prediction step leads to a system that contains as many unknown resistances as equations. However, this system may not be invertible. Thus, an extra step called resistance removal, is proposed so as to get an invertible extraction. For reasons detailed in Remark 2, the resistance removal step is performed before the minimization problem. The identification problem can then be solved, outputting a constriction prediction for the unknown set (see subsection 2.3). This is the inversion step. From there, plugged airways i.e. these with severe constrictions, are identified. See Figure 1 for a block flow diagram describing the whole process. Note that in this study, 4D ventilation data are synthetic and obtained by numerical simulation of the dynamical behavior of the tree-parenchyma coupled model presented in [15] (see subsection 2.2), to which some noise is added so as to reproduce realistic measurements.

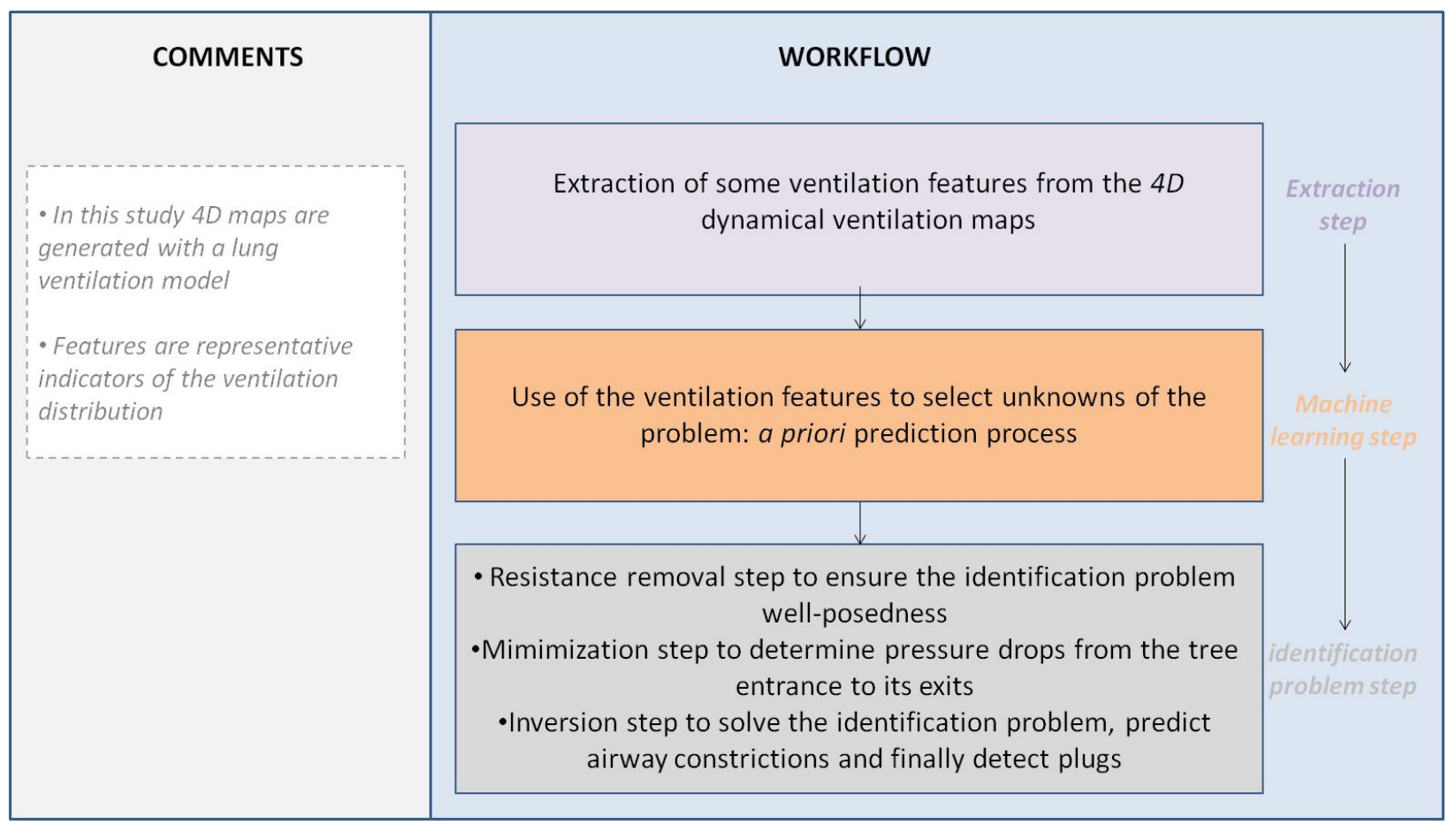

Figure 1: global process to determine the plug distribution in the tree based on dynamic ventilation data.

In the next section, the ventilation model is described.

\subsection{Direct ventilation problem}

Models for both the parenchyma and tree used in this study as well as the coupling between them are precisely described in [15].

Under tidal breathing, Mach numbers in the tree are low [20] and the flow is assumed to be incompressible, and it is reasonable to consider airways, the tree branches, as rigid cylindrical pipes [15]. Each branch is defined by its length $L$ and radius $r$. Viscous and inertial effects induce energy dissipations. The pressure drop $\Delta p$ and the flow $q$ in an airway are linked through a non linear coefficient $R(q)$, called resistance, by $\Delta p=R(q) q$. This coefficient is defined as in [21] by the Pedley model [22]: 


$$
R(q)=\left\{\begin{array}{lc}
\gamma\left(\operatorname{Re} \frac{2 r}{L}\right)^{\frac{1}{2}} R_{\text {pois }}, & \gamma\left(\operatorname{Re} \frac{2 r}{L}\right)^{\frac{1}{2}}>1 \\
R_{\text {pois }}, & \gamma\left(\operatorname{Re} \frac{2 r}{L}\right)^{\frac{1}{2}}<1
\end{array}\right.
$$

where $\gamma=0.327, R e$ is the Reynolds number defined by $R e=\frac{2 \rho|q|}{\mu_{f} \pi r}$ with $\rho$ the fluid density, $\mu_{f}$ its dynamic viscosity and the laminar Poiseuille resistance is $R_{p o i s}=\frac{8 \mu_{f} L}{\pi r^{4}}$. The term $\gamma\left(\operatorname{Re} \frac{2 r}{L}\right)^{\frac{1}{2}}$ is associated with inertial losses.

Remark 1: note that equation (2) has been designed for inspiration and for one bifurcation angle only. Following [23] we use it independently of the angle and we choose to also use it during expiration.

The parenchyma is a porous medium. Following [24], we treat it as a homogenized isotropic media occupying a domain $\Omega$, which is the configuration at rest. In tidal breathing conditions, i.e. breathing at rest with normal lung expansion, displacements are slow and with limited amplitude. We thus assume it follows a linear elastic Hooke's law. The related stress tensor is given by:

$$
\sigma_{m a t}(\boldsymbol{u})=2 \mu \varepsilon(\boldsymbol{u})+\lambda \operatorname{tr}(\varepsilon(\boldsymbol{u})) I
$$

where $\lambda$ and $\mu$ are the effective Lamé parameters, $\boldsymbol{u}$ is the parenchyma displacement defined in the reference state domain $\Omega$, and $\varepsilon$ the linear strain tensor defined by $\varepsilon(\boldsymbol{u})=\frac{1}{2}\left(\nabla \boldsymbol{u}+{ }^{T} \nabla \boldsymbol{u}\right)$.

If an airway collapses, its radius is decreased inducing a steep rise in resistance following equation (2). Whatever the elastic properties of the downstream region, it is more energy consuming and hence more difficult for the patient to push or pull flow through this branch. To take this phenomenon into account, the tree and parenchyma have to be coupled. In the following, to model the closure of an airway branch, we homogeneously reduce its radius.

In [15] we strongly couple the tree and parenchyma. Assuming $\Omega=\bigcup_{i=1}^{N} \Omega_{i}$ and $\forall i, j \Omega_{i} \cap \Omega_{j}=\emptyset$ where $\Omega_{i}$ is the sub-region of $\Omega$ irrigated by the $i^{t h}$ tree exit $e x_{i}$ (see Figure 2), and neglecting gravity, we obtain the following strong formulation:

$$
\operatorname{div}\left(\sigma_{m a t}\right)-\nabla p_{\text {tree }}=\rho_{\text {par }} \frac{\partial^{2} \boldsymbol{u}}{\partial t^{2}}, \text { in } \Omega
$$

Here $\rho_{\text {par }}$ is the parenchyma density and $p_{\text {tree }}$ is the piecewise constant function defined by:

$$
p_{\text {tree }}=\sum_{i=1}^{N}\left(p_{\text {trachea }}-(A Q)_{i}\right) \mathbb{1}_{\Omega_{i}}
$$

where $\mathbb{1}_{\Omega_{i}}$ is the characteristic function on $\Omega_{i}$, and $p_{\text {trachea }}$ is pressure at the trachea entrance. Vector $Q$ is defined by $Q=\left(q_{i}\right)_{1 \leq i \leq N}$ with $q_{i}$ the flow at the $i^{t h}$ exit. Note that under the assumption of small displacement around the reference state, we have

$$
q_{i}=q_{i}(\dot{\boldsymbol{u}})=\int_{\partial \Omega_{i}} \dot{\boldsymbol{u}} \cdot \boldsymbol{n}
$$

expressing that the volume variation of a given parenchyma region equals the volume of air entering or leaving that region. Thus, $p_{\text {tree }}$, and hence equation (3), can be written as functions of the displacement field only. 
Matrix $A$ includes information on airway resistances and thus on their dimensions. We denote $T_{i}$ as the set of indices of airways going from the trachea down to the $i^{t h}$ exit $\forall i \in \llbracket 1 ; N \rrbracket$. Set $T_{i}$ is defined by:

$$
T_{i}=\{(n, k) / \text { branch }(n, k) \in \text { path from the trachea down to exit } i\}
$$

and thus $T_{i}$ defines a unique path. Index $n$ refers to the airway generation starting at zero for the trachea. Index $k$ orders the airways of a given generation. A tree is said to be complete if each path contains the same number of generations. On a complete tree, at a given generation $n$, the index $k$ runs through the set $\llbracket 0 ; 2^{n}-1 \rrbracket$. From a complete tree one can extract a dyadic subtree as illustrated on Figure 2. Denoting $T_{i j}$ the intersection set between $T_{i}$ and $T_{j}$ we have $A=\left[A_{i j}\right]_{1 \leq i, j \leq N}$ with

$$
A_{i j}=\sum_{(n, k) \in T_{i j}} R_{n, k}
$$

with $R_{n, k}$ the resistance of branch $(n, k)$ (see Figure 2). These resistances are time dependent (see equation (2)).

Finally, the tree induces on each terminal region $\Omega_{i}$ an apparent pressure $p_{\text {tree }} / \Omega_{i}$ which opposes volume variations (see Figure 2). The greater the resistance on the path, going from the trachea down to exit $i$ denoted $e x_{i}$, the greater the pressure drop along this path, and the greater the apparent pressure. In case of airway closure, the fed related region expands or contracts at the price of an increased energy cost [5]. In the following, without loss of generality, we assume the pressure at the trachea is equal to zero. Dirichlet boundary conditions are prescribed on the parenchyma surface $\partial \Omega$, the surface displacement being given by $\boldsymbol{u}=\boldsymbol{u}_{\boldsymbol{D}}$ on $\partial \Omega$. The variational formulation associated with equation (3) is: for any $\boldsymbol{w}$ sufficiently smooth such that $\boldsymbol{w}=0$ on $\partial \Omega$,

$$
\rho_{\text {par }} \int_{\Omega} \ddot{\boldsymbol{u}} \cdot \boldsymbol{w}+\int_{\Omega} \sigma_{m a t}(\boldsymbol{u}): \varepsilon(\boldsymbol{w})-\int_{\Omega} p_{\text {tree }}(\dot{\boldsymbol{u}}) I: \varepsilon(\boldsymbol{w})=0,
$$

In the following (see subsection 2.3), $p_{\text {tree }}$ will be determined from the knowledge of $\boldsymbol{u}$. From there, we will develop insight on $A$ through equation (4). Note that, if the resistance distribution is known, $\boldsymbol{u}$ can then be determined by solving the direct problem defined by equation (7): this is how the synthetic ventilation $4 D$ maps are generated (see subsection 2.4.2). 


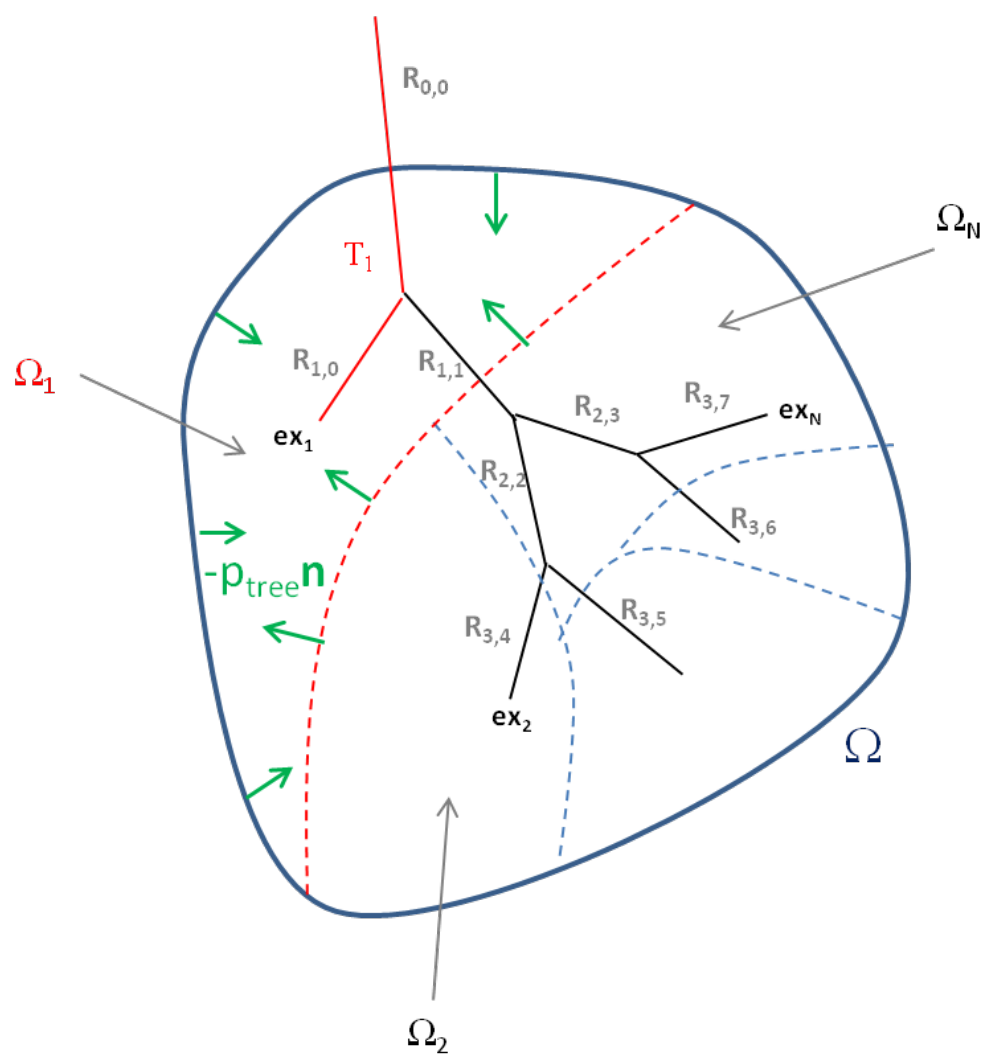

Figure 2: Schematic of parenchyma subdivision in non-intersecting regions $\Omega_{i}$, each one being irrigated by a tree path $T_{i}$. Representation on a non-complete dyadic tree. Each airway $(n, k)$ has a resistance $R_{n, k}$. Green arrows illustrate the apparent pressure $p_{\text {tree }}$ exerted by the tree through the coupling on region $\Omega_{1}$ when it expands.

Equation (7) is discretized in time (see [15]) through the scheme:

$$
\left\{\begin{array}{c}
\boldsymbol{u}^{n+1}=\boldsymbol{u}^{n}+\Delta t \boldsymbol{k}_{\boldsymbol{u}}^{n+1} \\
\dot{\boldsymbol{u}}^{n+1}=\dot{\boldsymbol{u}}^{n}+\Delta t \boldsymbol{k}_{\boldsymbol{v}}^{n+1} \\
\boldsymbol{k}_{\boldsymbol{u}}^{n+1}=\dot{\boldsymbol{u}}^{n}+\Delta t \boldsymbol{k}_{\boldsymbol{v}}^{n+1}=\dot{\boldsymbol{u}}^{n+1}
\end{array}\right.
$$

Space discretization is performed in the finite element (FE) framework, with Lagrangian P1 finite elements. We obtain the following linear system:

$$
E Y^{m+1}+B^{T} P^{m+1}=F^{m+1}
$$

with $P^{m+1}$ given by

$$
P^{m+1}=A^{m} B Y^{m+1}
$$

Vector $Y$ is the discrete FE representation of the velocity field $\dot{\boldsymbol{u}}, E$ stands for the FE mass and elastic matrices and depends on the parenchyma mechanical properties. The right hand side $F$ contains terms relative to surface displacement and velocity, thus depending on $\boldsymbol{u}_{\boldsymbol{D}}$ and $\dot{\boldsymbol{u}}_{\boldsymbol{D}}$. Details can be found in [15]. Matrix $B$ is defined by $B=\left[\int_{\Omega_{i}} \operatorname{div}\left(\boldsymbol{e}_{\boldsymbol{j}}\right)\right]_{1 \leq i \leq N, 1 \leq j \leq n_{\text {dof }}}$ where $\boldsymbol{e}_{\boldsymbol{j}}$ is the $j^{\text {th }}$ element of the finite element basis and $n_{\text {dof }}$ the number of finite element degrees of freedom of the problem; it links the discrete velocity field and the flow distribution $Q$ through

$$
B Y^{m+1}=Q^{m+1}
$$


As mentioned in subsection 2.1, some airways can be constricted in asthmatic lungs, from numerous moderate closures to a few severe constrictions. As in [5], based on a literature review, we propose to simulate asthma remodeling by including these two types of constrictions with stochastic laws. To simulate a broncho-constriction, the airway radius is homogeneously divided by a factor so-called constriction ratio. To model asthma, moderate constriction ratios are generated according to a log-normal law with average $\log (1.2)$, standard deviation 0.3 and within bounds $[1 ; 3]$. In addition, $n_{\text {closure }}$ severe constrictions are imposed, $n_{\text {closure }}$ being a random integer. These plugs are applied at randomly chosen positions of the tree and have constriction ratios randomly selected with a uniform law in the interval $[4 ; 10]$.

Some lung regions irrigated by constricted paths may be poorly ventilated, as seen with 4D-CT [11] and 4DMRI [3] images. Knowing the tree structure with constrictions' severity and position, which is information included in matrix $A$, the parenchyma mechanical properties $\rho_{p a r}, \lambda, \mu$, and the parenchyma surface displacement field over time $\boldsymbol{u}_{\boldsymbol{D}}$, one can compute the displacement field $\boldsymbol{u}$ within the lung and deduce the flow distribution within the tree. Though, the positions and severity of closures in the tree as well as mechanical properties are not a priori known. Some high-resolution imaging techniques make it possible to identify constrictions in proximal airways, typically only through generation 5 or 6 [7]. In the frame of this study, we assume that the lung parenchyma mechanical properties are known. This is not the case in practice but some techniques such as magnetic resonance elastography [25] may give relevant estimations. As described in subsection 2.1, we propose to use dynamic ventilation data to obtain the constriction distribution and to identify plugged airways. This approach will be referred to as the identification problem.

\subsection{Identification problem}

The function $p_{\text {tree }}$ is linked to the flow distribution $Q$ through the resistance matrix $A$ (see equation (4)). If both $p_{\text {tree }}$ and $Q$ were known, one could get insights on matrix $A$; thus yielding airway resistances (see equation (6)) and finally airway radii through equation (2). This identification step is done following a threestep approach. Image analysis on $4 D$ data gives access to the parenchyma displacement field $\boldsymbol{u}$ (see subsection 2.4.1) from which $Q$ can be computed given a parenchyma partition (5). Knowing the displacement field $\boldsymbol{u}$ along the breathing cycle, and assuming that both the parenchyma mechanical properties and the tree topology are known, one can use the ventilation model described in the previous subsection to define a function $p_{\text {tree }}^{*}$ satisfying a minimization problem. This is the minimization step. Pressure drops from the tree entrance down to the tree exits are linked to the flows within the tree through airway resistances, and the related system contains as many resistances as there are airways within the tree. However, from the knowledge of $Q$ and $p_{\text {tree, }}^{*}$, there is not enough information to recover every airway resistance. Based on the process described in this subsection, the number of unknowns is reduced so as to get an invertible system. This is the resistance removal step. In a last step, this system is inverted and airway radii are determined. This is the inversion step where plugged airways are identified.

\section{Minimization step:}

From the knowledge of the displacement field $\boldsymbol{u}$ and assuming that the mechanical properties of the parenchyma are known, we define a tree pressure $p_{\text {tree }}^{*}$ that is piecewise constant on each terminal region $\Omega_{i}$. The idea, based on the direct problem (3), is to minimize the distance between $\boldsymbol{\nabla} p^{*}$ tree and $\mathfrak{D}=$ $\operatorname{div}\left(\sigma_{\text {mat }}\right)-\rho_{\text {par }} \frac{\partial^{2} u}{\partial t^{2}}$ which is known by assumption. After time discretization, this minimization problem at each time step formally reads:

$$
\left\{\begin{array}{c}
\min _{p_{\text {tree } \in K_{i_{0}}}}\left\|\boldsymbol{\nabla} p_{\text {tree }}^{*}-\mathfrak{D}_{d}\right\|, \\
K_{i_{0}}=\left\{p_{\text {tree }}^{*}=\sum_{i} p_{\text {tree } / \Omega_{i}} \mathbb{1}_{\Omega_{i}} / p_{\text {tree } / \Omega_{i_{0}}}^{*}=p_{i_{0}}\right\}
\end{array}\right.
$$


where $i_{0}$ and $p_{i_{0}}$ are a given region index and the corresponding pressure drop, $\mathfrak{D}_{d}$ is a time discretization of $\mathfrak{D}$. Note that we have prescribed the value of $p_{\text {tree }}^{*}$ on the region $\Omega_{i_{0}}$ to ensure the uniqueness of $p_{\text {tree }}^{*}$ which is otherwise defined up to an additive constant. Note also that $\mathfrak{D}$ depends on the measurement of $\boldsymbol{u}$ as well as on the mechanical model that is chosen to describe the parenchyma behavior. The measures and the chosen model inaccuracies are two possible sources of error. After discretization, we get the discretized related minimization problem $\left(\mathcal{P}_{d}\right)$ defined at each time step $m$ by:

$$
\left(\mathcal{P}_{d}\right):\left\{\begin{array}{c}
\min _{P^{* m_{G}} \in \mathbb{K}_{i_{0}}}\left\|B^{T} P^{* m}-S^{m}\right\|^{2} \\
\mathbb{K}_{i_{0}}=\left\{V \in \mathbb{R}^{N} / V_{i_{0}}=p_{i_{0}}\right\}
\end{array}\right.
$$

where $P_{i_{0}}^{* m}$ is the $i_{0}{ }^{t h}$ component of $P^{* m}$ and $S^{m}$ is defined following (8) by

$$
S^{m}=F^{m+1}-E Y^{m+1} .
$$

Note that $B^{T}$ is the FE operator corresponding to the gradient operator acting on piecewise constant functions on the partition $\left(\Omega_{i}\right)_{i}$. Since $\operatorname{dim}\left(\operatorname{ker}\left(B^{T}\right)\right)=1$ (see Appendix A.1), $P^{* m}$ is defined up to a constant vector. This is the reason why a constraint on $P_{i_{0}}^{* m}$ is added. For the sake of clarity, temporal indices are removed in the following equations. The pressure constraint is enforced as described in subsection 3.2 by penalization.

To solve $\left(\mathcal{P}_{d}\right)$, the pressure drop $p_{i_{0}}$ along one path $T_{i_{0}}$ has to be known. Which path to use is determined based on the a priori prediction process described in subsection 2.4. Along this path we suppose airway dimensions are known and thus airway resistance (see Remark 2). To get the pressure drops through each airway, flows shall also be known (see equation (2)). Since the gas is incompressible and because airways are assumed to be rigid, the flow in branch $(n, k)$ equals the sum of the flows in its daughters:

$$
q_{n, k}=q_{n+1,2 k}+q_{n+1,2 k+1}
$$

So the determination of exit flows (see subsection 2.4.1) enables by recursion to assess flows in every airway. Knowing airway dimensions and flows along $T_{i_{0}}$, we can finally compute $p_{i_{0}}$ as $p_{i_{0}}=$ $-\sum_{(n, k) \in T_{i_{0}}} R_{n, k} q_{n, k}$.

Note that the minimization problem $\left(\mathcal{P}_{d}\right)$ is solved at each time step. Knowing flows and pressures, we now can seek the resistance distribution.

\section{Resistance removal step:}

In the following, we look for a resistance distribution such that vectors $A Q$ and $P$ are close (see equation (1)) where both $P$ and $Q$ are known. This leads to a linear system for which resistances $R_{n, k}$ are the unknowns. Once they are determined, since flows and airway lengths are supposed to be known, equation (2) gives the radii $r_{n, k}$ from which we can deduce the radii reduction ratios defined by

$$
\operatorname{red}_{n, k}=\frac{r_{n, k}}{r_{\text {healthy }},}
$$

and finally identify plugged airways. The smaller the reduction ratio, the more closed the airway. The pressure drop along any path $T_{i}$ is given by:

$$
\sum_{(n, k) \in T_{i}} R_{n, k} q_{n, k}=-p_{i} .
$$


The tree contains $2 N-1$ airways with $N$ exits. Thus the system (13) has size $N$ and contains $2 N-1$ unknowns. It is not invertible, thus the number of unknowns has to be reduced. The a priori prediction process described in subsection 2.4 provides for each airway a prediction of the radius reduction ratio (12) seen as a "constriction likeliness", based on which airways can be sorted. The lower the likeliness, the closer the airway is to its healthy configuration. From this ranking, the $N$ unknown resistances associated to the airways that are most likely to be plugged are kept, the $N-1$ airways associated to the remaining unknowns are assumed to have a healthy radius. At this stage, system (1) contains as many unknowns as equations. However, there is no guarantee that these equations will be independent. In practice, the system is invertible if the pressure at the nodes of unknown resistances are known, i.e. $R_{n, k}$ can be determined if $p_{n, k}$ and $p_{n-1,\left[\frac{k}{2}\right]}$ are known. Notation $[k]$ refers to the integer part of $k$.

Let $U_{N}$ be the set of indices of airways for which resistances are unknown. For each path $T_{i}$ from the tree mother branch to its $i^{\text {th }}$ exit, let us denote $T_{i_{N}}^{u}=T_{i} \cap U_{N}$. Then, $\forall i \in \llbracket 1 ; N \rrbracket$, (13) writes:

$$
\sum_{(n, k) \in T_{i_{N}}^{u}} R_{n, k} q_{n, k}=-p_{i}-\sum_{(n, k) \in T_{i} \backslash T_{i_{N}}^{u}} R_{n, k} q_{n, k} .
$$

Let us set a new indexation for airways with an unknown radius. We denote $\zeta_{N}$ a bijective function that maps elements of $U_{N}$ to the integer interval $\llbracket 1 ; N \rrbracket$ based on the ranking provided by the $a$ priori prediction process described in subsection 2.4: index 1 is associated to the airway that is predicted with the highest radius reduction ratio, i.e. the least likely to be constricted, and so on till index $N$ associated to the airway predicted with lowest radius reduction ratio. By extension, $\forall(n, k) \in U_{N}$ we note $R_{n, k}=R_{i}$ with $i=$ $\zeta_{N}(n, k)$ and $q_{n, k}=q_{i}$ (see Figure 3).

Let $Z_{N}$ be the matrix of $\mathcal{M}_{N}(\mathbb{R})$ defined by:

$$
Z_{N_{i j}}=\left\{\begin{array}{cc}
1 & \text { if } \zeta_{N}^{-1}(j) \in T_{i_{N}}^{u} \\
0 & \text { else. }
\end{array}\right.
$$

A given line $i$ of $Z_{N}$ contains 1 at position $j$ if the two following conditions are satisfied: $R_{j}$ belongs to the set of unknown resistances, and the airway associated to $R_{j}$ belongs to path $T_{i}$; otherwise the line contains 0 at position $j$.

We also define the vectors $G_{N}=\left(R_{i} q_{i}\right)_{i=1, \ldots, N}$ and $H_{N}=\left(-p_{i}-\sum_{(n, k) \in T_{i} \backslash T_{i_{N}}^{u}} R_{n, k} q_{n, k}\right)_{i=1, \ldots, N}$. With these notations system (13) is equivalent to:

$$
Z_{N} G_{N}=H_{N}
$$

Information on airway resistances and hence on their radii is contained in $G_{N}$. One has to solve system (15) to recover the resistances knowing the right hand side $H_{N}$. However, matrix $Z_{N}$ may not be invertible. The strategy is thus to extract from $Z_{N}$ an invertible matrix, which will be of maximal rank. To decide which resistance shall be removed from the unknowns, we define an iterative process taking advantage of the $a$ priori prediction made on the likeliness constriction of a branch. The higher the predicted reduction ratio for an airway, the less likely this airway is constricted. At iteration 1 the resistance $R_{1}$ of the airway with highest radius reduction ratio is removed with highest priority and the radius of the associated airway is set as "healthy". This corresponds to removing the first column of $Z_{N}$. The resulting matrix is not squared and contains at least two collinear rows, otherwise $Z_{N}$ would have been invertible (see demonstration in Appendix A.2); to keep a square system, one of these collinear rows is removed, defining matrix $Z_{N-1}$. Vectors $G_{N}$ and $H_{N}$ are modified accordingly, defining $G_{N-1}$ and $H_{N-1}$. If $\operatorname{rank}\left(Z_{N-1}\right)=N-1, Z_{N-1}$ is invertible and unknown resistances can be determined. If $\operatorname{rank}\left(Z_{N-1}\right)=\operatorname{rank}\left(Z_{N}\right)<N-1, Z_{N-1}$ is not invertible and we proceed to iteration 2. Otherwise, in case $\operatorname{rank}\left(Z_{N-1}\right)<\operatorname{rank}\left(Z_{N}\right), R_{1}$ is set back among the unknowns and the second airway resistance that is less likely to be plugged, $R_{2}$, is removed; the 
process goes on till $\operatorname{rank}\left(Z_{N-1}\right)=\operatorname{rank}\left(Z_{N}\right)$. Then the algorithm moves to iteration 2 and so on. Doing so, airways predicted as severely constricted by the a priori prediction process are less likely to be removed from the unknowns. Note that for reasons explained in Remark 2, we enforce the resistance of airway $(0,0)$ among the unknown set and prevent it from being removed. The precise description of the algorithm is given in Appendix A.3. Figure 3 gives a schematic illustration of the process.

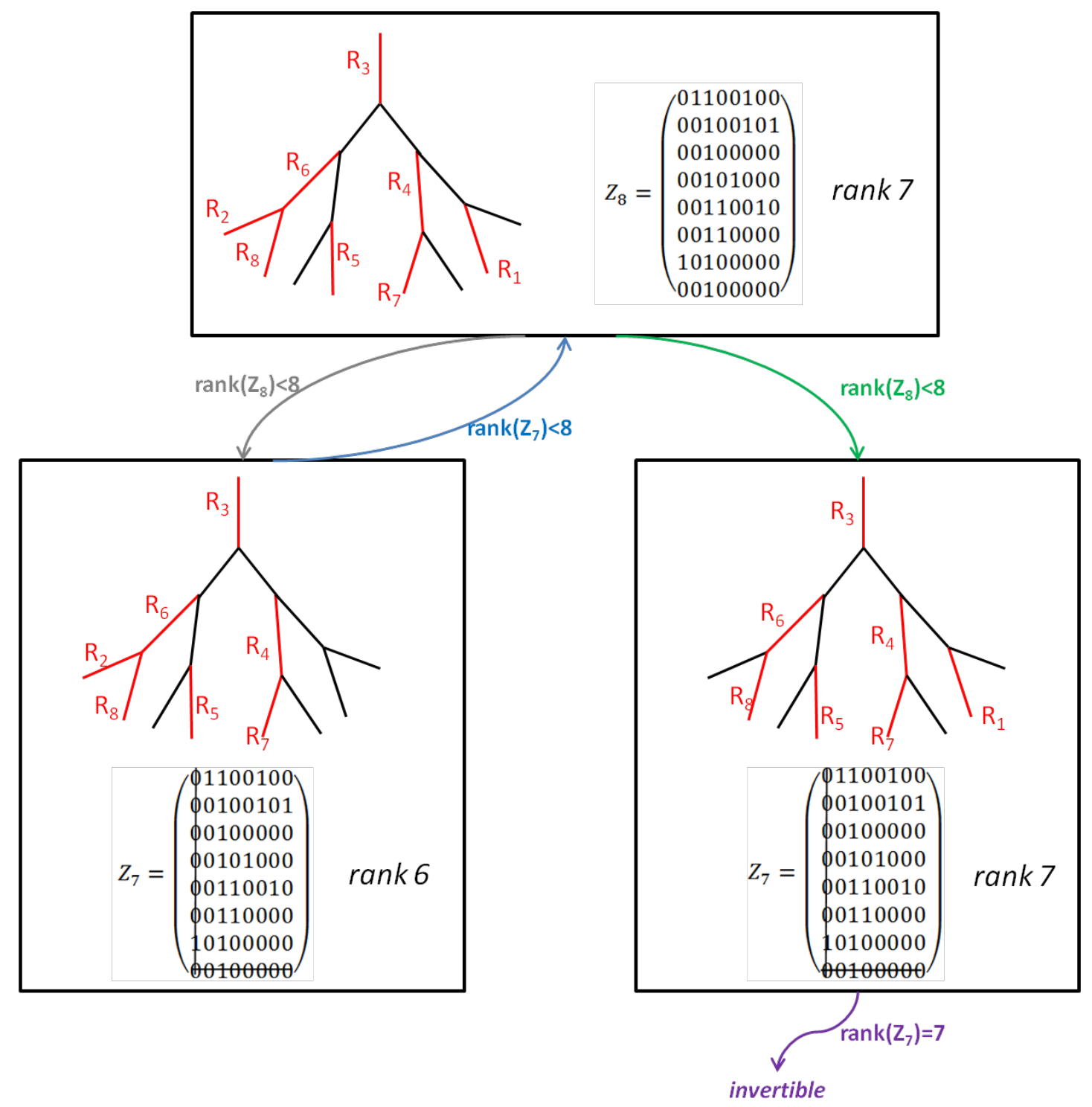

Figure 3: illustration of the resistance removal algorithm on an eight-exit tree. Branches in red are the ones with unknown resistance. Matrix $Z_{8}$ of the initial system is not invertible (top square). Following the process described in Figure $18, R_{1}$ is first removed from the set of unknowns, a line and a column are removed from $Z_{8}$ leading to $Z_{7}$ (bottom left square); rank $\left(Z_{7}\right)<$ $\operatorname{rank}\left(Z_{8}\right)$ so $R_{1}$ is set back among the unknowns (top square) and $R_{2}$ is removed instead (bottom right square). The resulting reduced matrix $Z_{7}$ now has the same rank as $Z_{8}$ so the removal is confirmed. At the end of the process the tree contains several paths with $R_{0,0}\left(=R_{3}\right)$ as only unknown, so a pressure along a path can be computed so as to solve the minimization problem (10), see Remark 2.

\section{Inversion step:}

If $Z_{l}$ is invertible we have, from (15), $G_{l}=Z_{l}^{-1} H_{l}$ and since the flows in all airways are known we can then determine the unknown resistances. We have $\forall i \in \llbracket 1 ; l \rrbracket, G_{l_{i}}=R_{i} q_{i}$. Then the radii can be determined from the Pedley formula (2), and 


$$
r_{i}=\left\{\begin{array}{cc}
{\left[\frac{q_{i}}{G_{b_{i}}} \frac{8 \mu_{f} L}{\pi}\right]^{\frac{1}{4}},} & \text { if } \gamma\left(\frac{4 \rho\left|q_{i}\right|}{\mu_{f} \pi L}\right)^{\frac{1}{2}}<1, \\
{\left[\frac{q_{i}}{G_{b_{i}}} \gamma 16\left(\frac{\rho \mu_{f}\left|q_{i}\right| L_{i}}{\pi^{3}}\right)^{\frac{1}{2}}\right]^{\frac{1}{4}},} & \text { else. }
\end{array}\right.
$$

Recall that for each airway with a resistance in the unknown set, a radius is computed at each time step, and the final prediction is taken as the time average of these values. Note that the resistance removal step is performed only once; thus the resulting invertible matrix $Z_{l}$ does not depend on the time step.

In practice, most of the airways are moderately constricted. Assuming they are healthy will therefore induce some errors in the right hand side $H_{l}$ and thus some prediction errors on the unknowns since they will have to account for a higher pressure drop on their paths.

Remark 2: To solve equation (10), the pressure drop along a path has to be imposed. To compute the pressure drop $p_{i_{0}}$ along a path $T_{i_{0}}$, radii of airways belonging to $T_{i_{0}}$ must be known (recall that lengths and flows are supposed to be known). After the a priori detection process, to make sure that at least one path contains airways with known dimensions only, the resistance of airway $(0,0)$ is enforced among the unknowns of system (13) and prevented to be removed during the resistance removal step (described in Figure 18). Doing so, one path $T_{i_{0}}$ of the tree will contain no other unknown than airway $(0,0)$. Indeed, if $R_{0,0}$ is enforced among unknowns, one has to access $p_{1,0}$ to ensure invertibility. To that end, there needs to be a path with no unknown from node $(1,0)$ to an exit. In practice, airway $(0,0)$ would be the trachea. Its dimensions can be measured (with bronchoscopy for example) and enforcing $R_{0,0}$ among unknowns is a technique designed to enable $p_{i_{0}}$ computation. Thus, dimensions of airways along $T_{i_{0}}$ are all known and the pressure drop $p_{i_{0}}$ along this path can be computed. Note that the minimization step is therefore performed after the resistance removal step.

Remark 3: Note that by solving an extracted system from (15) we do not ensure system (13) is satisfied. To verify system (13) some compatibility conditions on the right hand side would be needed. The last invertible extracted system $Z_{l} G_{l}=H_{l}$ could be enriched by adding unknown resistances while keeping the system invertible. But our first aim here is not to get a resistance distribution that allows for the recovery of the displacement field measured on the $4 \mathrm{D}$ data. The goal of this study is rather to identify plugged airways. Note however that if the resistance of an airway that is actually plugged is removed from the set of unknowns, it will not be identified as a plug.

To summarize, the identification problem aims at determining which airways are severely constricted. It consists of three steps and is performed after the a priori detection process. Pressure drops are linked to the flows within the tree through airway resistances by system (13). This system contains more unknown resistances than independent equations. The unknown set has to be reduced, this is the resistance removal step, which is performed one time and is valid for every time step. The removal step ensures the existence of a path through which the pressure drop along the tree can be computed. The minimization problem $\left(\mathcal{P}_{d}\right)$ can then be solved to determine the pressure drops from the tree entrance to all its exits. Now that exit pressures are known, the system extracted from (13) is inverted at each time step.

As mentioned before, a selection process is needed to determine which resistance to remove from the unknown set. This process shall keep, among others, the airways that are actually plugged and remove some healthy or moderately constricted airways, i.e. some that have negligible effect on ventilation distribution. Indeed, as mentioned in subsection 2.1, asthma affects the lung structure from many moderate radius constrictions to a limited number of airway closures. Using the ventilation distribution information provided by dynamic images, we propose a methodology to make an a priori prediction on airways constriction likeliness (see subsection 2.4). 
Remark 4: We aim to keep in the unknown set resistances associated to the most constricted airways. If the a priori prediction process provides an appropriate ranking, airways whose resistances do not belong to that set are moderately constricted at most. Their radius is supposed to have a typical "healthy" value known from morphometric studies (see subsection 3.1.3), although normal airway dimensions are submitted to an inter- and intra- variability [19]. However, possible radius variation within this "healthy" or "normal" range are much lower than these induced by severe bronchoconstrictions. The errors caused by considering an arbitrary "healthy" radius are thus expected to have little impact on the pressure drop distribution compared to plugs.

\subsection{A priori prediction process}

Following the process described in subsection 2.3, to get insights on plugs distribution, only a sub-set of airway resistances can be considered as unknowns. From the $2 N-1$ airways, at most $N$ have their resistance initially set as unknown (see subsection 2.3). One could try all the combinations of $N$ airways such that system (15) is invertible, invert the system for each combination, and keep as a final result the one that minimizes the distance between vectors $A Q$ and $P$. However, it can be shown that there are more than $2^{N-1}$ of these combinations: this makes this approach costly when the tree contains a few hundred exits as done in this study. Thus, before the identification problem step, an a priori prediction process is required to determine which branches are likely to be constricted and which ones rather have close to healthy dimensions. These latter are removed with highest priority. The prediction process described in this section is based on dynamical ventilation data analysis. It uses a machine learning technique to map the observed ventilation distribution with constrictions positions and strengths within the tree.

\subsubsection{Ventilation information extraction}

Ventilation distribution, i.e. flows within the tree, is related to the tree structure. In the following, some features characterizing the ventilation are set as inputs of a machine learning algorithm so as to determine which airways are likely to be plugged. The ventilation distribution can be determined from the parenchyma velocity field. Indeed, from (5) one can compute tree exit flows. Under the incompressibility assumption, the flow distribution within the tree is then obtained (11).

\subsubsection{Machine learning process}

The machine learning process proceeds in two steps (see Figure 4). First a correspondence is established between ventilation features extracted from $4 D$ ventilation data and known airway radius reduction ratios (12) that are associated to these features. This mapping is built on a large synthetic database, called the learning base, so as to capture the diversity of ventilation distribution patterns. This is the learning step. In a second step, for a dynamical ventilation map associated to an unknown constriction distribution, ventilation features are extracted, and airway reduction ratios are determined. This is the target prediction step.

\section{Ventilation features:}

A key question to address is which ventilation features to consider in the machine learning process. Bronchoconstrictions influence the ventilation distribution. To quantify disease severity and to obtain insight on the geometrical distribution of constrictions, ventilation pathological patterns can be compared to the ones that would be obtained if there were no constrictions. In this study we assume that for a given asthmatic patient lung mechanical properties are known. Also known is the tree topology, i.e. nodes positions, and the typical dimensions of the airways if they were not constricted. This is the "healthy" tree 
configuration. We finally assume we can access the lung geometry and the parenchyma surface displacement during breathing. These are the necessary input data to use the tree-parenchyma coupled model described in subsection 2.2. A reference "healthy" ventilation distribution can thus be simulated along with various synthetic "asthma" cases.

The machine learning step aims at determining, given a ventilation distribution, which airways are likely to be constricted and with which severity. To do so, we need to define at the airway level some features that correlate with the ventilation distribution. These features are precisely defined in Appendix A.4. In particular, they enable to compare the "healthy" configuration to "asthmatic" ones (see the description of the database just below).

Once these features are defined we use a random forest regressor algorithm to map for each airway $(n, k)$ its feature vector (as defined in Appendix A.4) and its radius reduction ratio $\frac{r_{n, k}}{r_{h_{e a l t h y}, k}}$. Quality of the prediction results is shown in subsection 4.2. The random forest method was chosen because it is fast and insensitive to noise.

Remark 5: Note that ventilation distribution is also influenced by factors that do not appear among the selected features. In particular, it is a function of the position within the lung: for instance, in tidal breathing regime, displacements are more important around the diaphragm than near the lung apex. To make the prediction more accurate, one could add as features the lobar position of the airway, or even its exact position within the lung. Though doing so would dramatically increase the problem dimensionality. Rather than providing a precise prediction of the constriction ratio, we aim at predicting a good ranking of potentially plugged airways (see results subsection 4.2). For that reason, the output of the machine learning algorithm may be referred to as "constriction likeliness" rather than radius reduction ratio. Airways could have been sorted based on other criteria. For instance, one could have enforced proximal airways in the unknown set (see subsection 2.3 ) by modulating the prediction with the airway generation.

\section{Database construction and constriction likeliness prediction:}

To build a learning base the following approach is adopted: first the parenchyma displacement field is extracted from the $4 D$ dynamical map, so-called target map, associated to a tree whose plug distribution has to be determined. This would typically be the map obtained from an asthmatic patient, a so-called target patient. The extracted surface displacement is used as a set of Dirichlet boundary conditions (namely the surface displacement through time $\boldsymbol{u}_{\boldsymbol{D}}$ ). Knowing the tree structure, the lung mechanical properties, and assuming that the parenchyma behavior can be accurately described by a Hooke's law model, a healthy ventilation simulation is performed based on the tree-parenchyma coupled model described in subsection 2.2. Imposing stochastic constrictions distributions as described in subsection 2.2, synthetic pathological ventilations are simulated with the same given Dirichlet boundary conditions. Note that in practice, the parenchyma surface displacement may depend on the bronchoconstriction distribution. Here the database is built for the specific target patient. All synthetic patients breathe with the same surface displacement but various airway resistances. If the database is large enough, generated synthetic ventilation patterns allow the identification of the target patient plug distribution. From the synthetic data, airway ventilation features described in Appendix A.4 are computed. Note that the associated radius reduction ratios are known since the trees have been generated. The learning phase is performed on this synthetic database by mapping the features and the related known airway constrictions. If the tree contains $2 N-1$ airways and if $m_{p}$ pathological cases are simulated, there are $(2 N-1) * m_{p}$ couples (airway features; radius reduction ratio) in the learning base.

This database is then the basis to predict airway radius reduction ratios associated to the target $4 D$ dynamical map. As mentioned in subsection 2.1, in this study the target map is synthetically generated with the tree-parenchyma coupled model. 


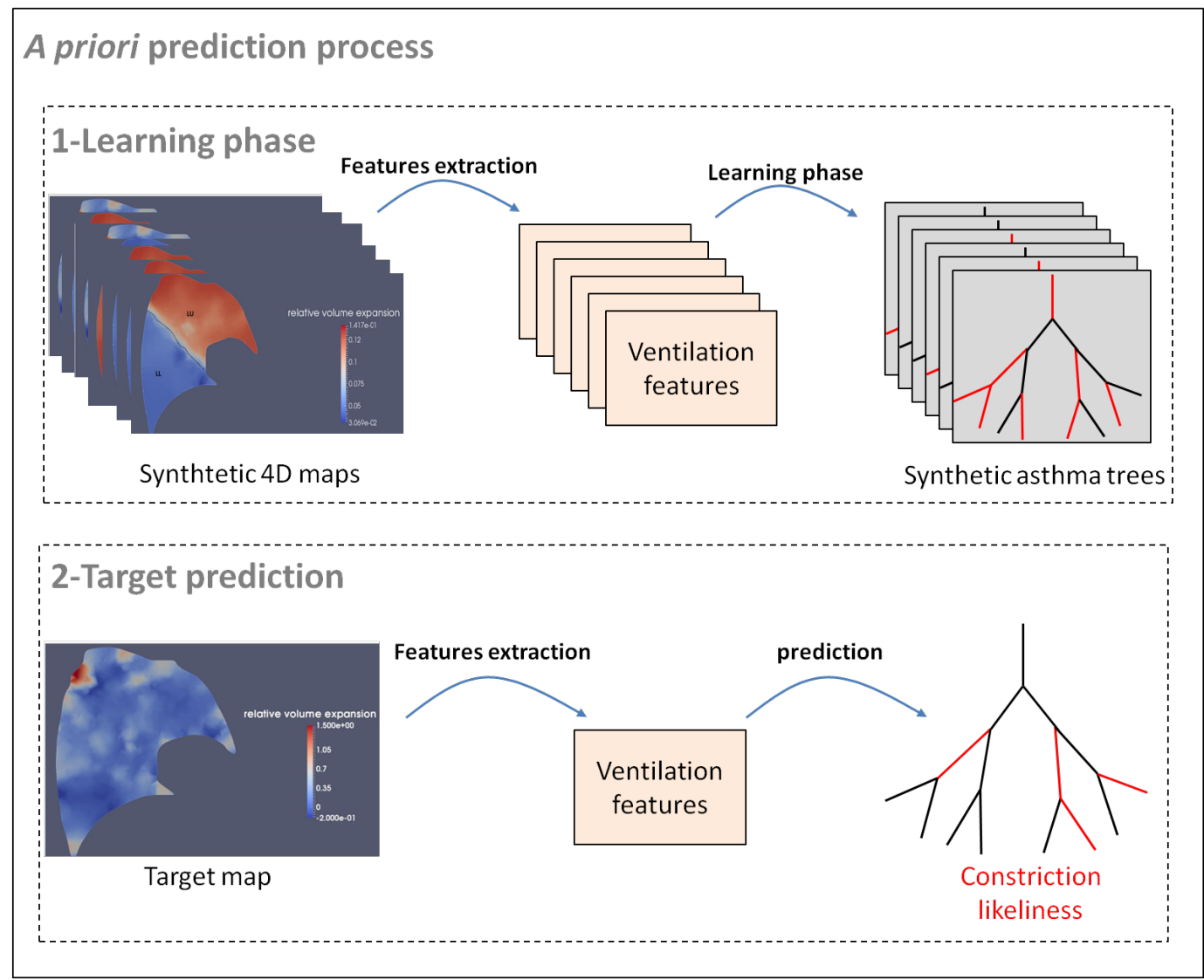

Figure 4: Schematic of the a priori prediction process. In the learning phase, a mapping is built between ventilation features extracted from synthetic $4 D$ ventilation data and airway constriction ratios in related asthmatic trees. Note that the Dirichlet boundary conditions and the tree geometry used to generate the synthetic data are those of the target ventilation map. Then, airway constriction likeliness is predicted for the tree associated to the target ventilation map. If the process is good, plugged airways (in red) have a high constriction likeliness.

\subsection{Global methodology summary}

In this section we summarize the overall process to go from a $4 D$ dynamical map and tree topology to the tree plugs distribution, see Figure 5. For the identification problem (step 4, see subsection 2.3) to be wellposed, not every airway resistance can be considered as an unknown. An a priori prediction process (step 3 ) is used to select a subset of airways that are more likely constricted and shall hence be put in the unknown set. Based on a machine learning technique (see subsection 2.4.2 and Appendix A.4) ventilation features extracted from the target ventilation map (step 1, see subsection 2.4.1) are used to predict constriction likeliness for each airway. To that end, a learning phase is necessary. To generate synthetic data for the learning phase, the tree-parenchyma ventilation model presented in [15] and subsection 2.2 is used. The extracted parenchyma surface displacement is used as a set of Dirichlet boundary conditions. Ventilation simulations are performed with a healthy tree model and various asthmatic configurations (step 2, see subsection 2.4.2). The healthy tree model is built following subsection 3.1.3; segmented branches are used along with the parenchyma envelope to generate complete tree geometry. Asthmatic configurations are obtained by imposing stochastic constrictions as described in subsection 3.1.3. Using the a priori constriction likeliness ranking, one can finally proceed to the identification problem step (step 4) which is done in three stages. The system linking flows and tree pressures through airway resistances is a priori not invertible. An invertible extraction is first obtained thanks to the resistance removal step. Then a minimization problem is solved to determine the exit tree pressures. The resulting system is inverted and the plug locations and severity are identified. 


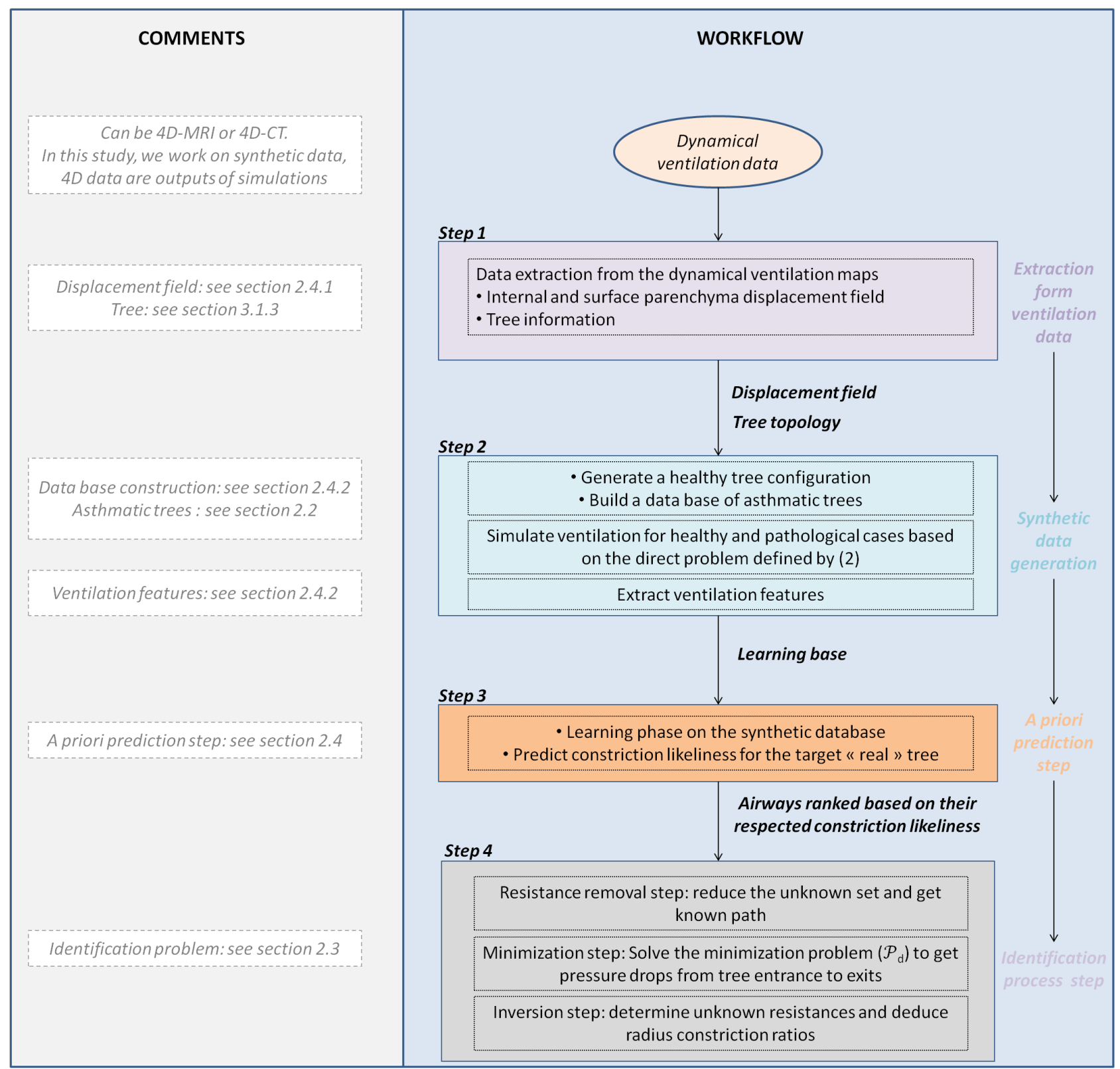

Figure 5: schematic of the overall process, from $4 D$ dynamical data to plugged airways identification. Detailed version of Figure 1.

\section{Numerical methods and patient-specific data exploitation}

In this section we describe patient-specific data construction, numerical strategies and systems resolution.

\subsection{Input data construction}

For the process described in Figure 5, the lung geometry, its displacement over the breathing cycle and the tree structure are needed. 


\subsubsection{Lung geometry}

From $4 D$ scans the lung envelope can be segmented. We work on one human left lung geometry. Segmented HRCT data acquired in [26] are used to build a patient-specific tree and a parenchyma geometry.

A surface mesh of the parenchyma is generated with Meshlab [27]. From there, a tetrahedral volume mesh is built with GMSH [28]. It is subdivided based on the tree structure in as many regions as there are terminal branches (see Figure 6) according to an algorithm described in [15]. The mesh contains 250594 tetrahedrons, which corresponds to achievable 4D-CT spatial resolution (see subsection 3.1.2). There are 140091 degrees of freedom.

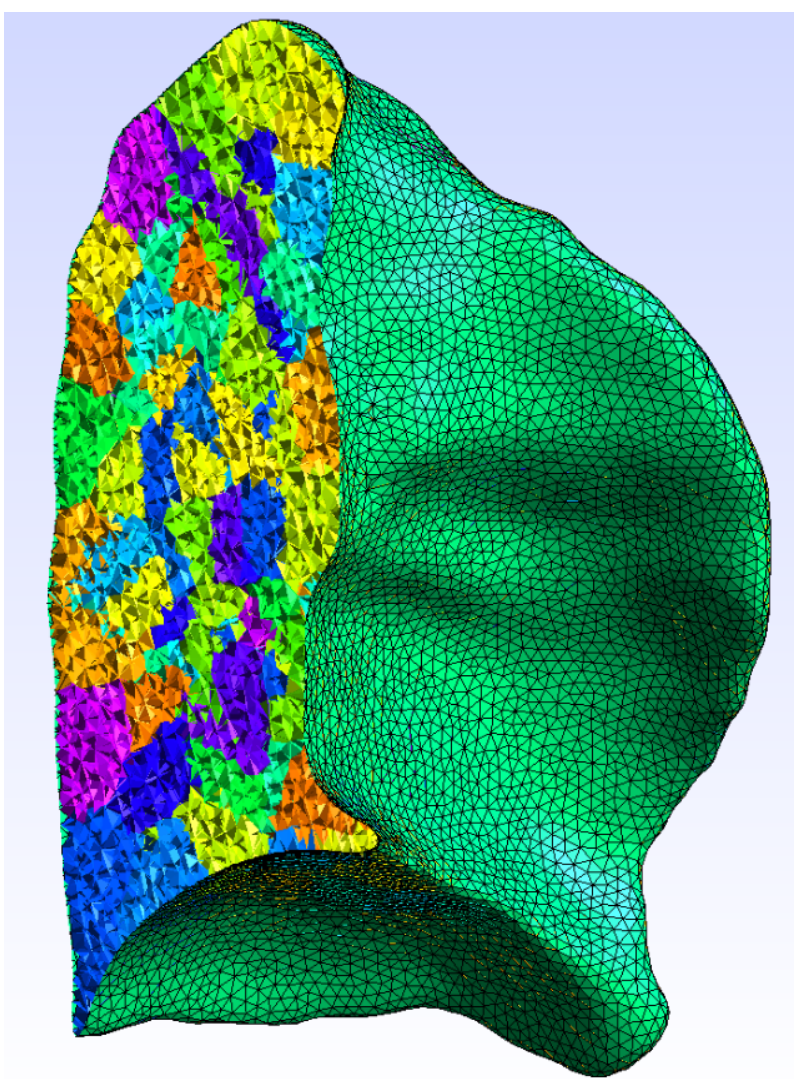

Figure 6: Left lung mesh subdivision based on a ten-generation tree structure. The domain is divided into 477 terminal regions. Each color corresponds to a different region. The average volume of each region at the reference state is $2.2 \mathrm{ml}$.

\subsubsection{Temporal and spatial resolutions}

Synthetic $4 D$ ventilation data generated from the tree-parenchyma coupled model described in subsection 2.2 are used in this study. Spatial and temporal resolutions are chosen consistent with what currently available machines can provide. Simulations performed to generate these data are done with a small time step of $0.04 \mathrm{~s}$ to ensure precision but only a few time points are then kept to cope with experimental temporal resolutions.

The parenchyma surface Dirichlet boundary condition is built based on two static inflation states segmented from [29]: mean lung volume (MLV), here considered as the reference state, and total lung capacity (TLC). A non-linear registration is performed with the software deformetrica [30] to map MLV and TLC parenchyma surfaces. In this study we consider tidal breathing conditions, i.e. for respiration at rest and with normal volume expansion. The MLV to TLC registered displacement field is thus bounded and sinusoidal dynamics with a time period of $4 \mathrm{~s}$ are prescribed so as to impose a typical tidal volume 
evolution. This surface displacement is imposed as Dirichlet boundary conditions to the tree-parenchyma coupled model. Here we work on synthetic data: the displacement field $\boldsymbol{u}(t)$ is the output of a simulation based on the mechanical model described in subsection 2.2. Note that, as mentioned in subsection 2.3 , one could work on real $4 D$ data from which a displacement field can be extracted through registration. Depending on the scanner and reconstruction techniques, 4D-CT and 4D-MRI lead to various spatial and temporal resolutions [31], [32], [33], [34]. We assume an isotropic spatial resolution of $4 \mathrm{~mm}^{3}$ and we use a mesh that is refined accordingly (see subsection 3.1.1). Temporal resolution is $0.4 \mathrm{~s}$ (i.e. ten images per breathing cycle in this study). Synthetic ventilation data are thus generated every $0.4 \mathrm{~s}$ to perform the machine learning step (see results in subsection 4.2). Data from the ten resulting time points are aggregated to proceed with the identification problem step (see results in subsection 4.3).

Whatever the technique, registration is not exempt from errors. In the following we conduct the identification process both on noise-free and noisy data. Based on [31], [32], [33], noisy data are generated by multiplying at each time step $m$ each component $i$ of $Y^{m}$, the FE approximation of the velocity field at time $m \Delta t$, by a factor $\left(1+\theta_{i}^{m}\right)$ where $\theta_{i}^{m}$ is generated according to a uniform law in the interval $[-0.3 ; 0.3]$. This corresponds to an uncorrelated noise going up to $\pm 30 \%$. Note that in practice, 4D-CT data can exhibit strong artifacts leading to gross errors in the computed displacement [35]. The methodology described in this paper assumes synthetic data are free from such artifacts.

\subsubsection{Tree structure}

From $4 D$ scans, upper airways can be segmented and used along with the parenchyma surface to propagate a conductive tree model with a patient-specific space filling approach [36], see Figure 7.

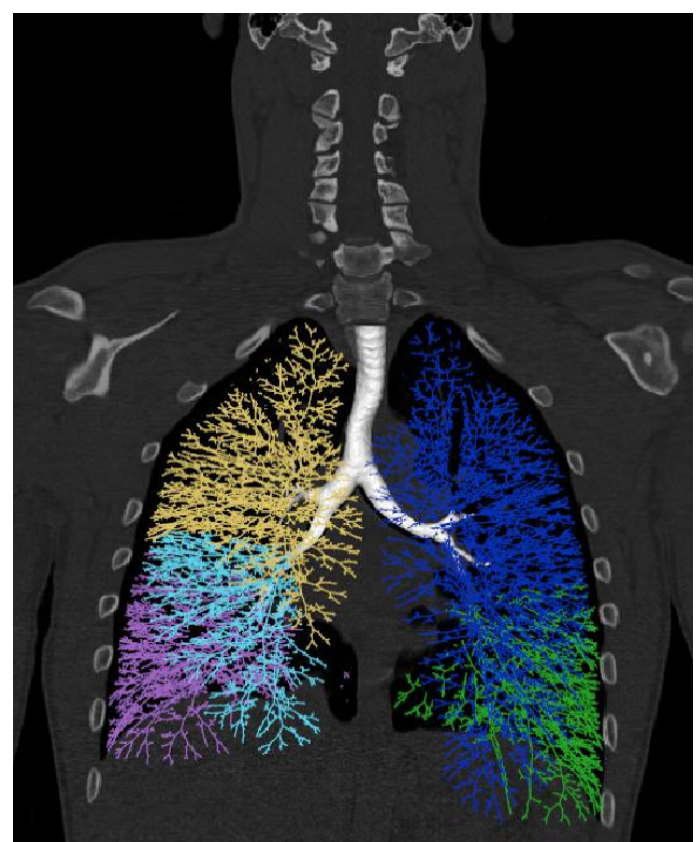

Figure 7: Space filling tree obtained based on the work presented in [36]. Segmented upper airways and lung envelope are used to propagate a patient-specific $\mathbf{O D}$ model of the conductive tree structure. Each color corresponds to a different lobe. Figure taken from [15].

The resulting structure contains a faithful representation for segmented upper branches, and a simulated tree representation model for the remaining airways. This model is built based on both general physiological knowledge and patient data; it generates nodes positions, airway lengths and radii. While constrictions may be seen on segmented upper airways [7], the propagated part of the tree does not account for any pathology. Indeed, there is no a priori knowledge on the distribution of constricted branches, which is specific to each patient. Node positions and airway lengths of the propagated part of the tree are thus from now on known. Airway radii $r_{h e a l t h y_{n, k}}$ are in a healthy configuration range (see Remark 4). Note that since the upper airway geometry is known from segmentation, their resistance would not 
need to be included among the unknowns of the identification problem described in subsection 2.3. Though, in this study, we work on synthetic pathological trees with stochastic constrictions generated by the process described in subsection 2.2; we consider all branches radii including proximal ones are $a$ priori unknowns.

In the following, we work on ten-generation space filling trees. Downstream sub-trees are treated as symmetric units and characterized by equivalent resistances. Each unit is irrigated by one airway referred to as a tree exit. Constrictions in such a subtree would be accounted for by a resistance increase of the related exit. The resulting left lung tree used herein contains 954 airways among which 477 are terminal branches. When simulating asthma, we generate $n_{\text {closure }}$ plugs with $n_{\text {closure }}$ randomly chosen in the interval $\llbracket 1 ; 40 \rrbracket$ (see subsection 2.1), this interval being set based on non-published data from [36].

\subsubsection{Mechanical properties}

In this study, the parenchyma mechanical properties are assumed to be homogeneous and are set within the ranges provided in [37]: Young's modulus $E=1256 \mathrm{~Pa}$ and Poisson ratio $v=0.4$ where $E$ and $v$ are linked to the Lamé parameters by $\lambda=\frac{v E}{(1+v)(1-2 v)}$ and $\mu=\frac{E}{2(1+v)}$. Parenchyma density is $\rho_{\text {par }}=$ $100 \mathrm{~kg} \cdot \mathrm{m}^{-3}$. Air density and viscosity at $37^{\circ} \mathrm{C}$ are respectively $1.125 \mathrm{~kg} \cdot \mathrm{m}^{-3}$ and $1.89510^{-5} \mathrm{~Pa} . \mathrm{s}$.

\subsection{Numerical methods}

\section{Direct problem:}

System (8) is solved through a conjugate gradient descent method with Jacobi preconditioning as described in [15].

\section{Machine learning:}

The machine learning step is performed with a random forest regressor. The number of elements in the forest is chosen to be 50 and other parameters (as proposed by the scikit-learn library [38]) are set to their default values. In order to predict the airway radius reduction ratios of a patient, synthetic data are generated as described in subsection 2.4.2 and used for the learning phase.

In this study, we work on one healthy tree and 49 asthmatic ones, the mechanical parameters and constitutive relation being the same in every cases. We adopt a leave-one-out strategy: iteratively, one of these pathological trees is considered as unknown in the sense that the aim is to predict the radius reduction ratios of its airways. The 48 other ones are used to build the learning base, which thus contains more than 45,000 airways. Prediction results are shown in subsection 4.2.

Airways are ranked based on their predicted ratios, and this ranking is used for the unknown selection (see subsection 2.3). As mentioned in subsection 3.1.2, the identification problem is performed on both noisefree and noisy synthetic ventilation data.

\section{Identification problem:}

The solution of the minimization problem $\left(\mathcal{P}_{d}\right)$ (see (10)) is approached by solving the penalization problem

$$
\min _{P \in \mathbb{R}^{N}}\left\|B^{T} P-S\right\|^{2}+\frac{1}{\varepsilon}\left(\Pi_{i_{0}}(P)-p_{i_{0}}\right)^{2}
$$

with $\varepsilon>0$ and small and $\Pi_{i_{0}}$ the linear form that maps a vector of $\mathbb{R}^{N}$ into its $i_{0}{ }^{\text {th }}$ component. Numerically, we solve

$$
B B_{\text {penalized }}^{T} P=B S_{\text {penalized }}
$$


where

$$
B B_{\text {penalized }}^{T}=B B^{T}+\frac{1}{\varepsilon} I_{i_{0}}
$$

with

$$
\left[I_{i_{0}}\right]_{i j}=\left\{\begin{array}{cc}
1, & \text { if } i=j=i_{0} \\
0, & \text { else. }
\end{array}\right.
$$

The penalized matrix $B B_{\text {penalized }}^{T}$ is invertible. Matrix $B S_{\text {penalized }}$ writes

$$
B S_{\text {penalized }}=B S+\frac{1}{\varepsilon} V_{i_{0}}
$$

with

$$
\left[V_{i_{0}}\right]_{i}=\left\{\begin{array}{cc}
\frac{1}{\varepsilon} p_{i_{0}}, & \text { if } i=i_{0} \\
0, & \text { else. }
\end{array}\right.
$$

We finally solve $B B_{\text {penalized }}^{T} P=B S_{\text {penalized }}$ with the conjugate gradient method. Then, as described in subsection 2.3, an extracted system of (15) is solved at ten time points during the breathing cycle (see temporal resolution in subsection 3.1.2). At each of these time points, radius reduction ratios of airways belonging to the unknown set are computed. If the average radius reduction ratio defined by (12) over the ten time points is below 0.3 , the airway is classified as a plug. This bound is set based on the radius reduction ratio distributions (see subsection 2.2). Some airways that are actually more severely constricted are indeed predicted as plugged (they are true positives), some are missed (they are true negatives), and some that are only moderately constricted are classified as plugged (thus they are false positive).

\section{Results}

In this section we present some results relative to the direct problem (see subsection 2.2), the machine learning step (see subsection 4.2) and the identification problem (see subsection 4.3) on a human left lung, which thus contains two lobes, the left upper lobe (LU) and the left lower lobe (LL).

\subsection{Results for the direct problem}

Here we present the results of two typical cases (for a more extensive description, see [15]): a healthy case and a configuration in which a plug with radius reduction ratio $1 / 7$ is applied on the branch that feeds lobe LU. Results shown here are plotted after a few cycles so that the periodically converged regime has been reached. In Figure 8 the volume expansion from the reference state is plotted for both cases. Recall that a Dirichlet boundary condition with a sinusoidal breathing cycle is imposed (see subsection 3.1.2). This prescribes the total lung volume variation, which is thus also the same in the pathological case; though the air distribution between both lobes is affected. In the constricted case, lobe LU is less ventilated in favor of lobe LL. Note also that, along the breathing cycle, LU does not expel all the gas it contains: there is air trapping which is typical of asthma and COPD [39]. Finally, due to the resistance increase, ventilation of the diseased region is shifted in time. This has been reported in [40]. In Figure 9 we plot the scalar field $\operatorname{div}(\boldsymbol{u})$ which represents the local relative volume expansion for the two cases. 


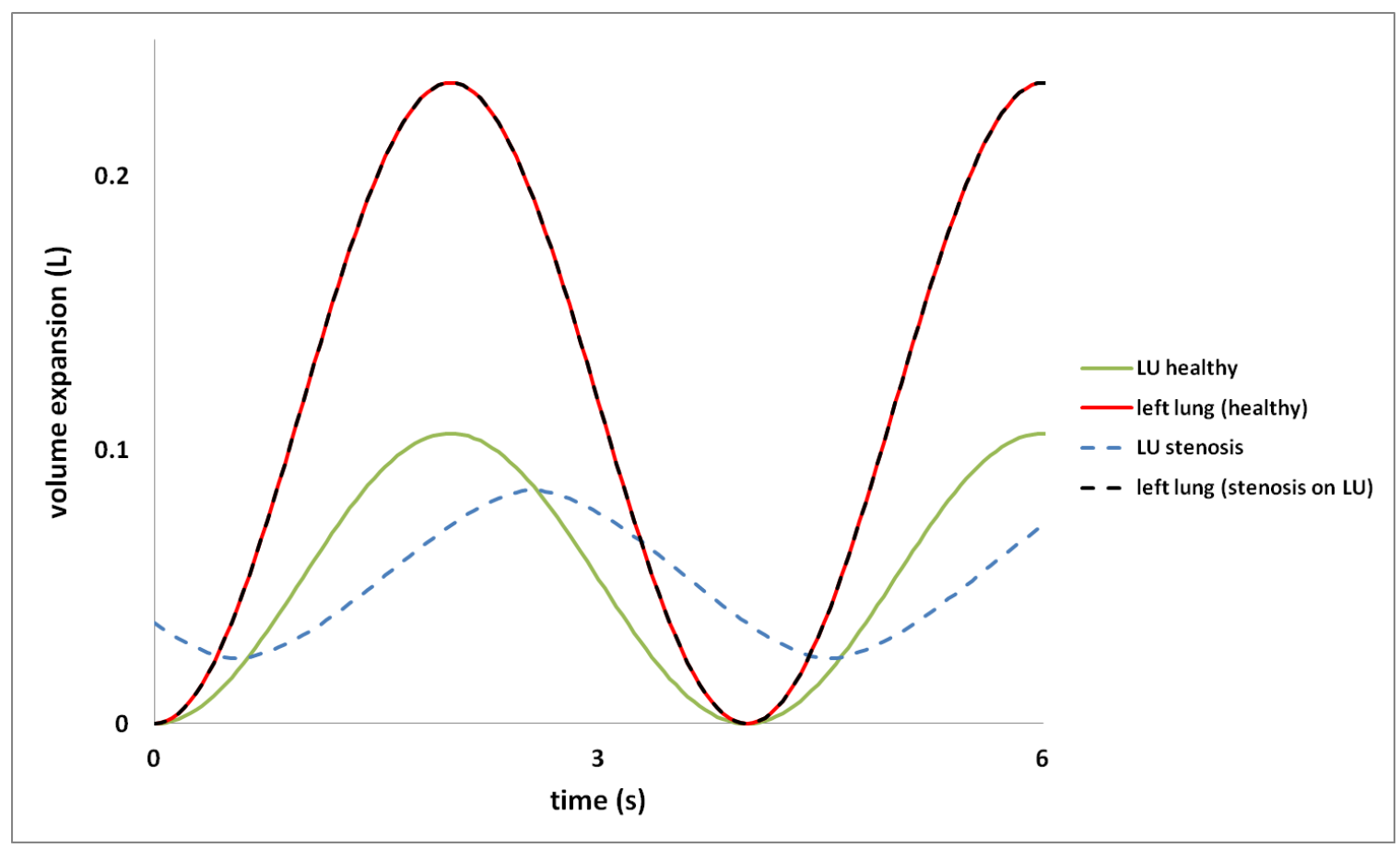

Figure 8: Left lung and LU lobe volume expansions from the reference state in a healthy configuration and when a bronchoconstriction, with constriction ratio 7, is applied to the branch that feeds lobe LU. Dirichlet boundary conditions with sinusoidal dynamics are applied to the system.

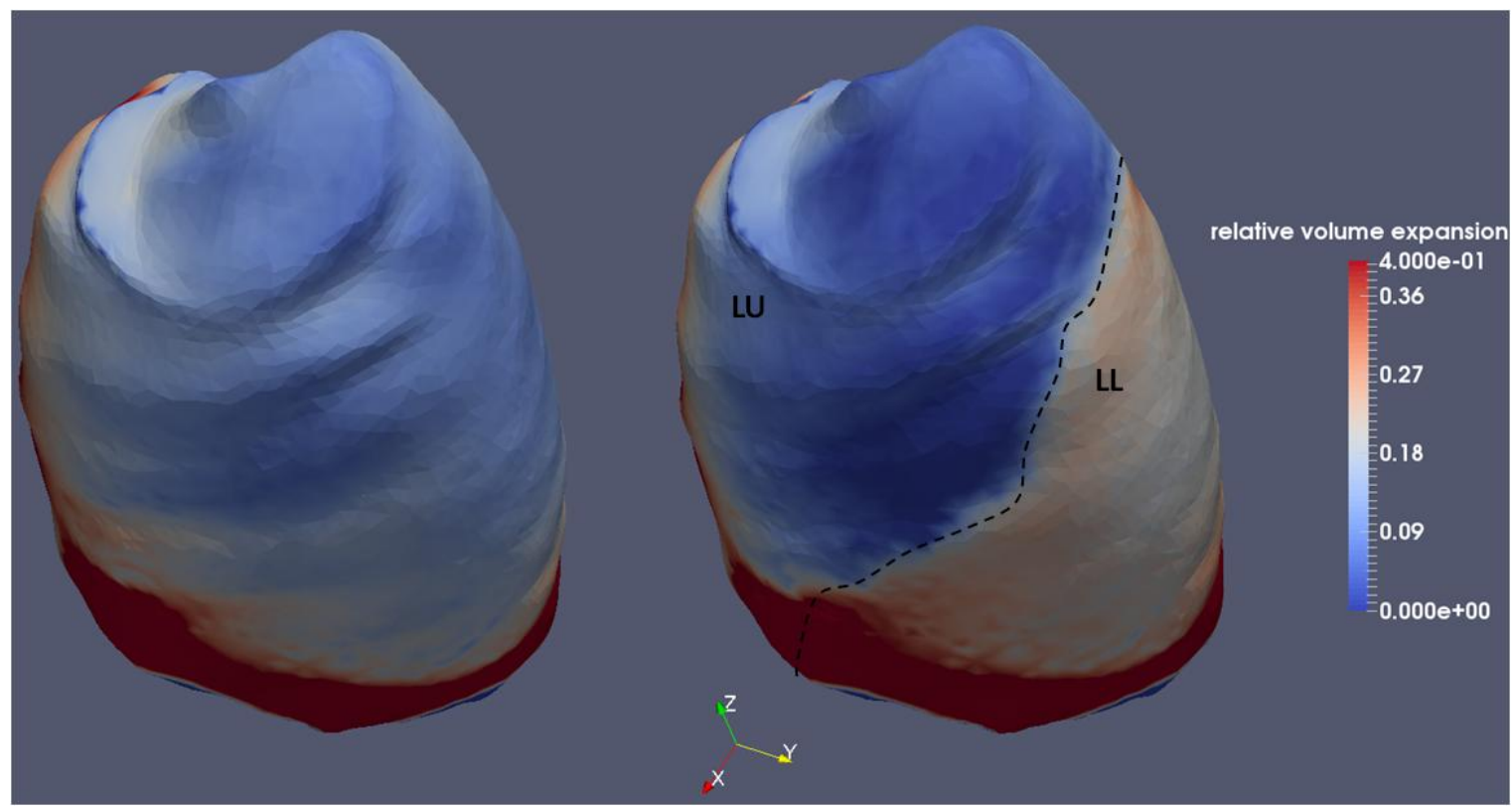

Figure 9: Local relative volume expansion 3D map on a left lung geometry. Dirichlet boundary conditions with sinusoidal breathing cycle are imposed. On the left side a healthy configuration is simulated. On the right side a bronchoconstriction with constriction ratio 7 is applied on the branch that feeds lobe LU. Plot at time=1s. Figure taken from [15]

Consistent with previous observations, lobe LU expands less in the pathological case. Note that diaphragmatic motion induces higher expansion in lower regions of the lung.

As explained in subsection 2.1, an asthmatic tree contains both a few severe constrictions and numerous moderate constrictions. Here we will compare the influence of moderate and severe bronchoconstrictions on ventilation distribution. To do so, we first build 49 stochastic asthmatic trees following the process described in subsection 2.2. Then we generate "plugs only" trees built from the asthmatic trees by removing moderate constrictions, and "plugs free" trees built from asthmatic trees by removing the plugs. We compare first the ventilation distribution obtained in the asthma and the "plugs free" cases, and then 
the ventilation distribution obtained in the asthma and the "plugs only" cases. In Figure 10, we plot for each of the 49 trees the ratio

$$
\operatorname{diff}_{\text {plugsonly }}=\frac{100}{N} \sum_{i=0}^{N}\left|\frac{\left|V_{i}^{\text {max }}-V_{i}^{\text {min }}\right|_{\text {onlyPlugs }}-\left|V_{i}^{\text {max }}-V_{i}^{\text {min }}\right|_{\text {asthma }}}{\left|V_{i}^{\text {max }}-V_{i}^{\text {min }}\right|_{\text {asthma }}}\right|
$$

where index $i$ refers to tree exit $i$. The sum is over the $N$ tree exit airways, here 477 , and notations $V_{\max }$ and $V_{\min }$ have been defined in subsection 2.4.2. Similarly we define dif $f_{\text {plugsFree }}$.

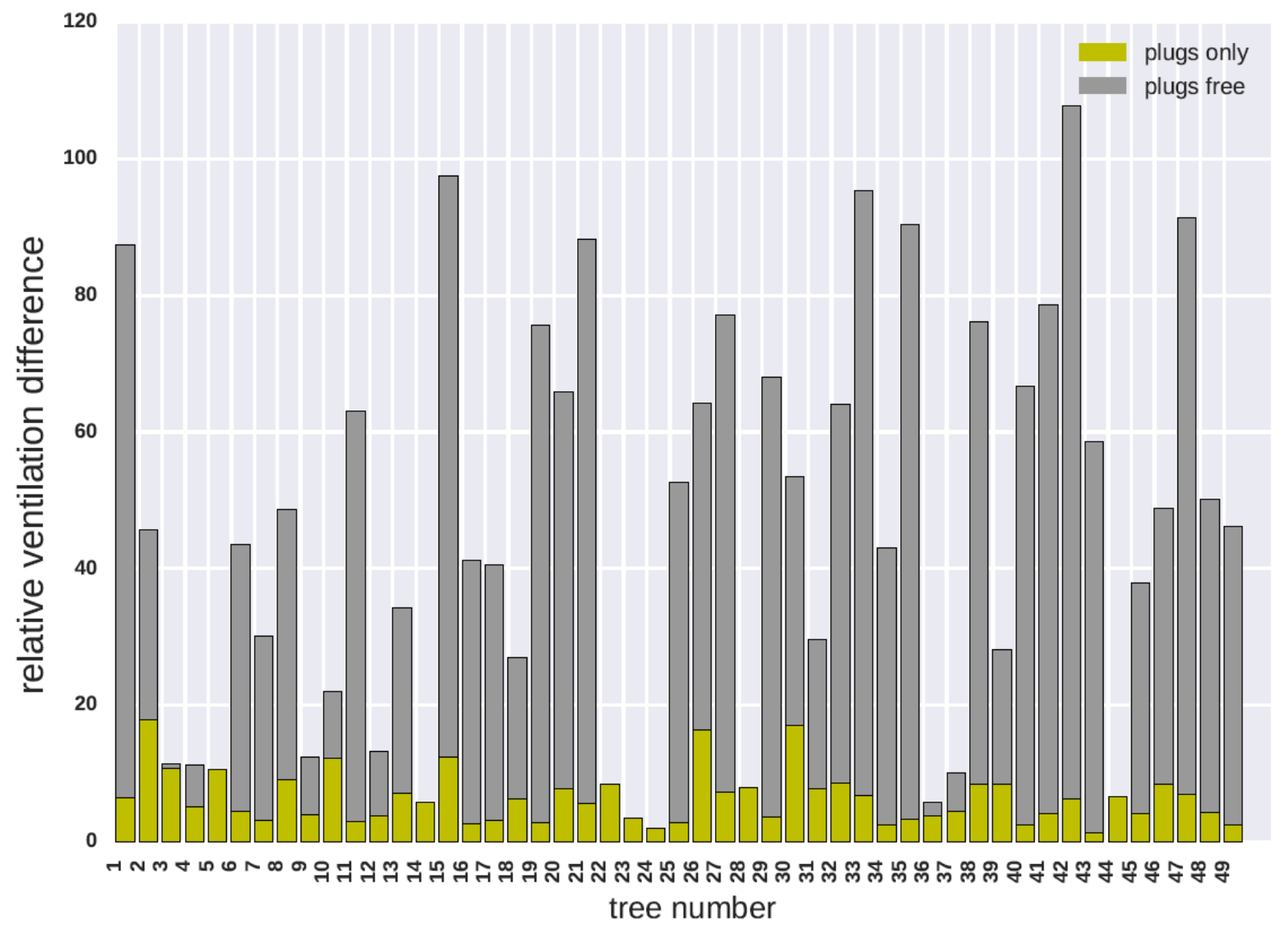

Figure 10: Plot of diff $_{\text {plugsonly }}$ (yellow) and diff $f_{\text {plugsFree }}$ (grey) for each of the 49 asthma trees. Note that these are relative differences with respect to the full asthma tree.

We observe in Figure 11 that in all cases the ventilation distribution obtained with plugs only is close to the one obtained with both plugs and moderate constrictions (6\% difference on average). In most cases there is a high discrepancy between the asthma and the "no plugs" ventilation ( $45 \%$ difference on average). As a conclusion, plugs are much more influential than moderate constrictions on ventilation distribution in this asthma model. In some cases (e.g., trees 14 or 22), plugs have little influence on the ventilation distribution. These are configurations in which the number of plugs is in fact low (see Figure 11). These data are the basis for determining the position and severity of constrictions based on $4 D$ ventilation data.

\subsection{Results for the machine learning step}

In this section, we assess the quality of the machine learning step described in subsection 2.4.2. As explained in subsection 2.3, not all tree resistances can be considered as unknowns of the process. First, among the 954 airways, $N=477$ have to be selected. A radius reduction ratio (which is in range [0;1], see equation (12)) is predicted for each airway and branches are sorted in increasing order. The smaller the 
ratio, the more likely the airway is constricted. In Figure 11, we plot, for each of the 49 synthetic asthma trees, the total number of plugs and among them the number for which the predicted radius reduction ratio is sorted among the first 477.

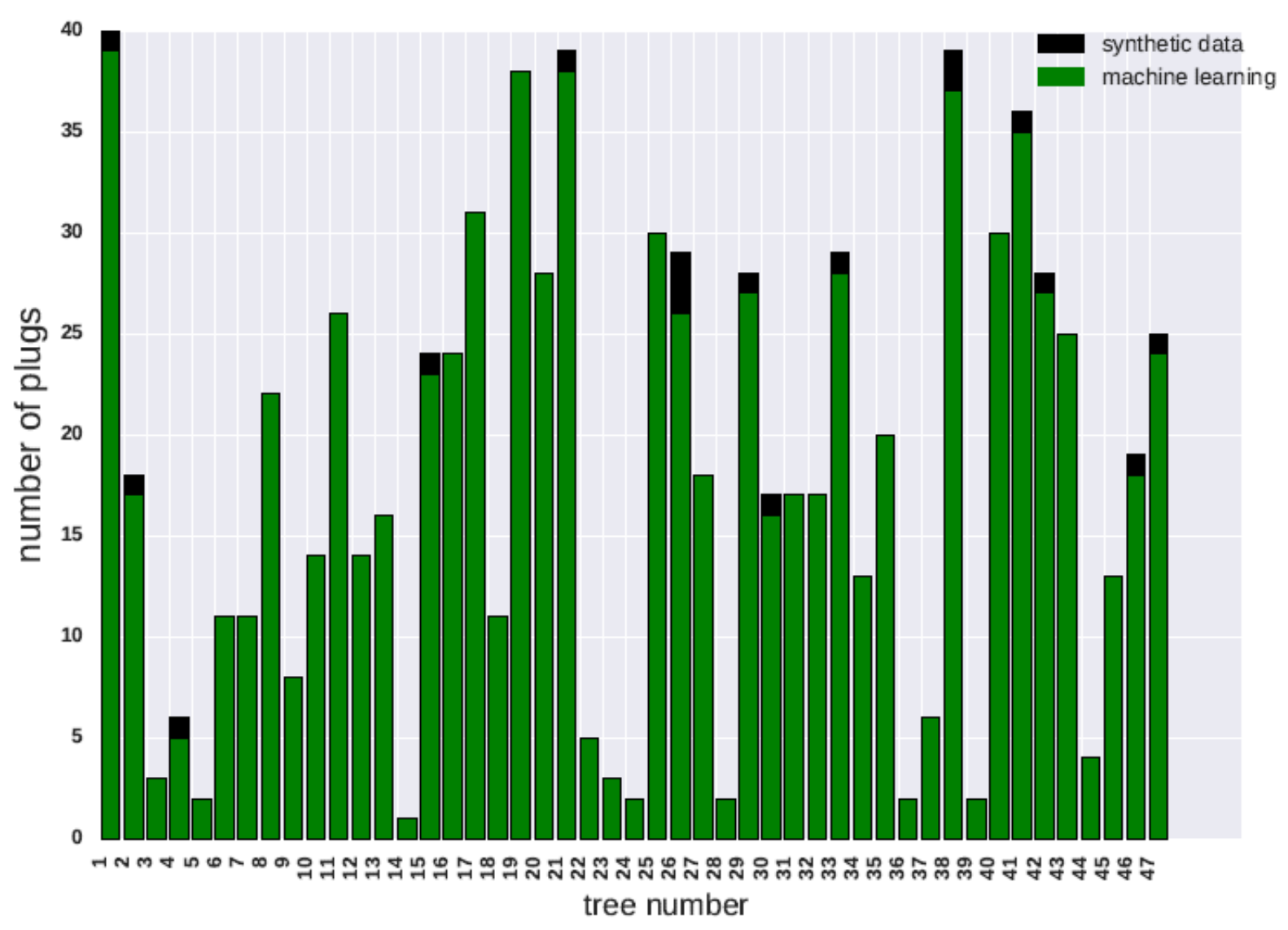

Figure 11: Machine learning classification success. The machine learning step predicts for each airway a radius reduction ratio. A low ratio corresponds to a severe constriction. Airways are sorted in increasing order with respect to their predicted ratio. Thus, each airway has a rank; the smaller the rank the smaller the predicted radius reduction ratio. For each asthmatic tree are plotted the total number of plugs (ground truth as they come from synthetic data), and among them the number of plugs that are classified with radius constriction ratio in the first half by the machine learning step. Trees contain 954 airways.

At this stage, for all trees, a large majority of the plugged airways if not all have their resistances kept in the unknown set, $98.5 \%$ in average (see Table 1). Among these $N$ unknowns, some will be removed to get an invertible extraction of system (15) (see subsection 2.3) based on the constriction likeliness ranking provided by the machine learning step. For an airway with rank $k$, we call $\frac{k}{N}$ its normalized rank. The smaller the normalized rank, the less likely the airway will be removed from the unknown set. The a priori prediction process is judged to be good when plugged airways have a low normalized rank and are thus likely to be kept in the final unknown set. Note that on average on the 49 trees, after the resistance removal process (see Figure 18), there are 250 kept unknowns over the 954 initial tree airways.

In Figure 12 we plot, for each tree, the average normalized rank of plugged airways obtained when ventilation features are extracted from noise-free and noisy $4 D$ ventilation data. Details on the noise structure can be found in subsection 3.1.2. Note that the average normalized rank depends on the number of plugs within the tree which goes from 1 to 40 (see subsection 3.1.3). If a tree contains one plug only, as for tree number 24, the best achievable average rank is obtained when the plug ranks first and its value is $1 / 954 \approx 0.1 \%$. If a tree contains 40 plugs, as for tree number 1 , the best achievable average rank is obtained when the plugs rank among the 40 first and its value is $20 / 954 \approx 2 \%$. 


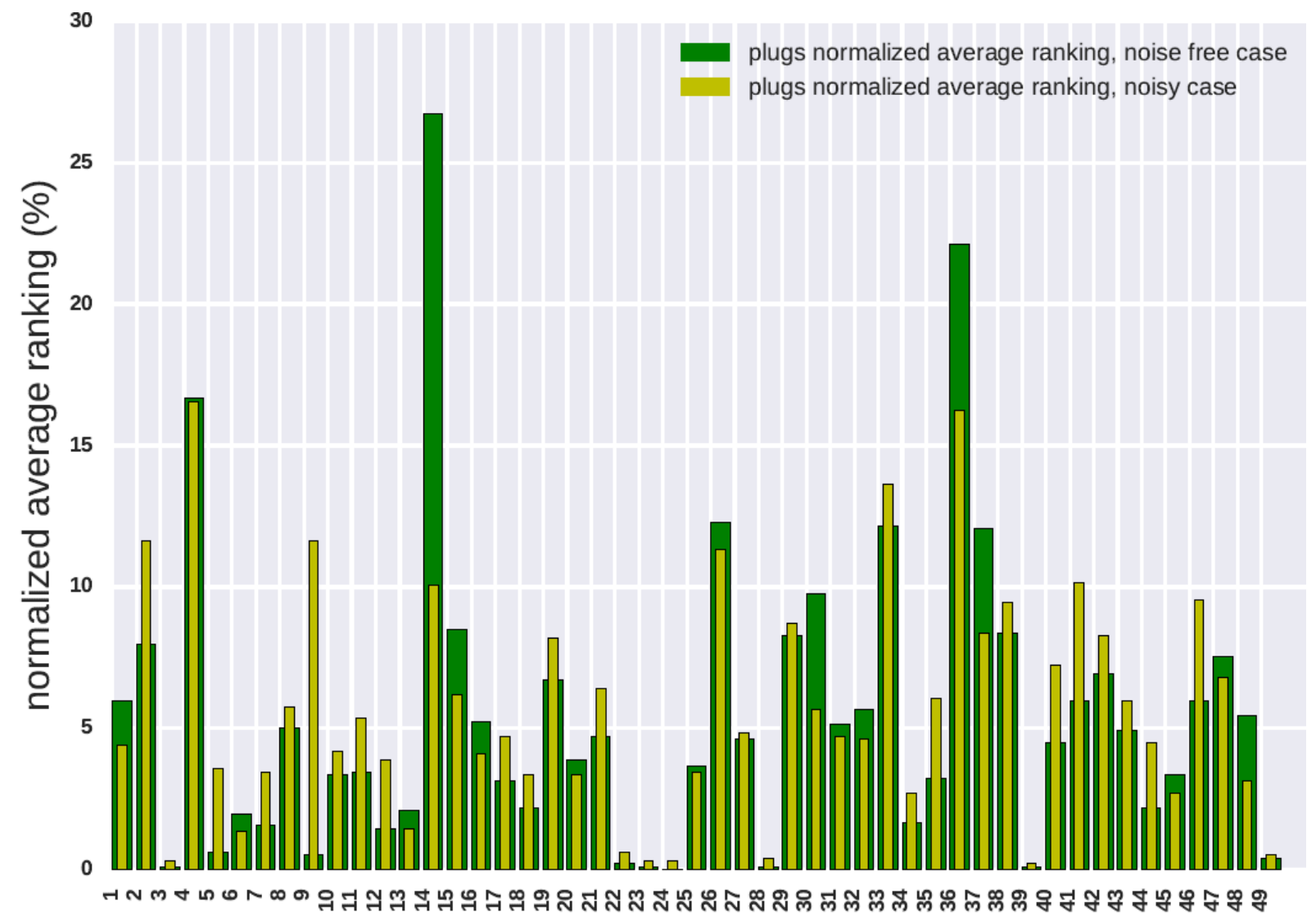

Figure 12: Normalized average ranking of plugged airways by the machine learning step. The machine learning step predicts for each airway a radius reduction ratio. A low ratio corresponds to a severe constriction. Airways are sorted in increasing order according to their predicted ratio. Thus, each airway has a rank; the smaller the rank, the smaller the predicted constriction ratio. The average predicted rank of plugged airways divided by 954 , the total number of airways, is plotted for each asthmatic tree. The lower this normalized average rank, the more likely resistances of plugged airways will be kept in the unknown set during the removal process (see subsection 2.3 ). In green (resp. yellow) results obtained when ventilation features are extracted from a noise-free (resp. noisy) $4 D$ ventilation data.

The machine learning step is consistent when applied to the cases with noise. In about $90 \%$ of the cases plugged airways are ranked in the first decile, for both the noise-free and noisy case. The average rank is good, thus airways that are actually plugged are not likely to be removed during the selection process described in Figure 3. Although the machine learning step provides good rankings, it does not accurately predict airways constriction. The identification problem is needed to determine which branches are actually plugged.

\subsection{Results for the identification problem step}

In this section are presented the results of the identification problem step (see subsection 4.3) without and with noise added to the velocity field coming from 4D data. As mentioned in section 2.1 the inputs come from synthetic data generated by the tree-parenchyma coupled model (see subsection 2.2). Simulations are performed over one breathing cycle. The identification problem classification process is performed as described in subsection 2.3.

In Figure 13, we plot for each asthmatic tree the number of plugged airways that are properly identified as plugged by the identification problem step divided by the total number of plugged airways, and the number of false positives divided by the total number of plugged airways. As explained in subsection 3.2, an airway is classified as plugged by the identification problem if its average computed radius reduction ratio is below 0.3. Most of the plugs are detected. In some cases, airways radius reduction ratios are overestimated by the identification problem step and they are not classified as plugged although they should be. For a few 
airways, the ratio is under-estimated and they are classified as plugged although they should not be. Thus, they appear as false positives. The identification problem detection rate quantifies the proportion of plugged airways that are properly classified after the identification problem step. The false positive detection rate quantifies the proportion of airways classified as plugs while they are actually moderately constricted at most.

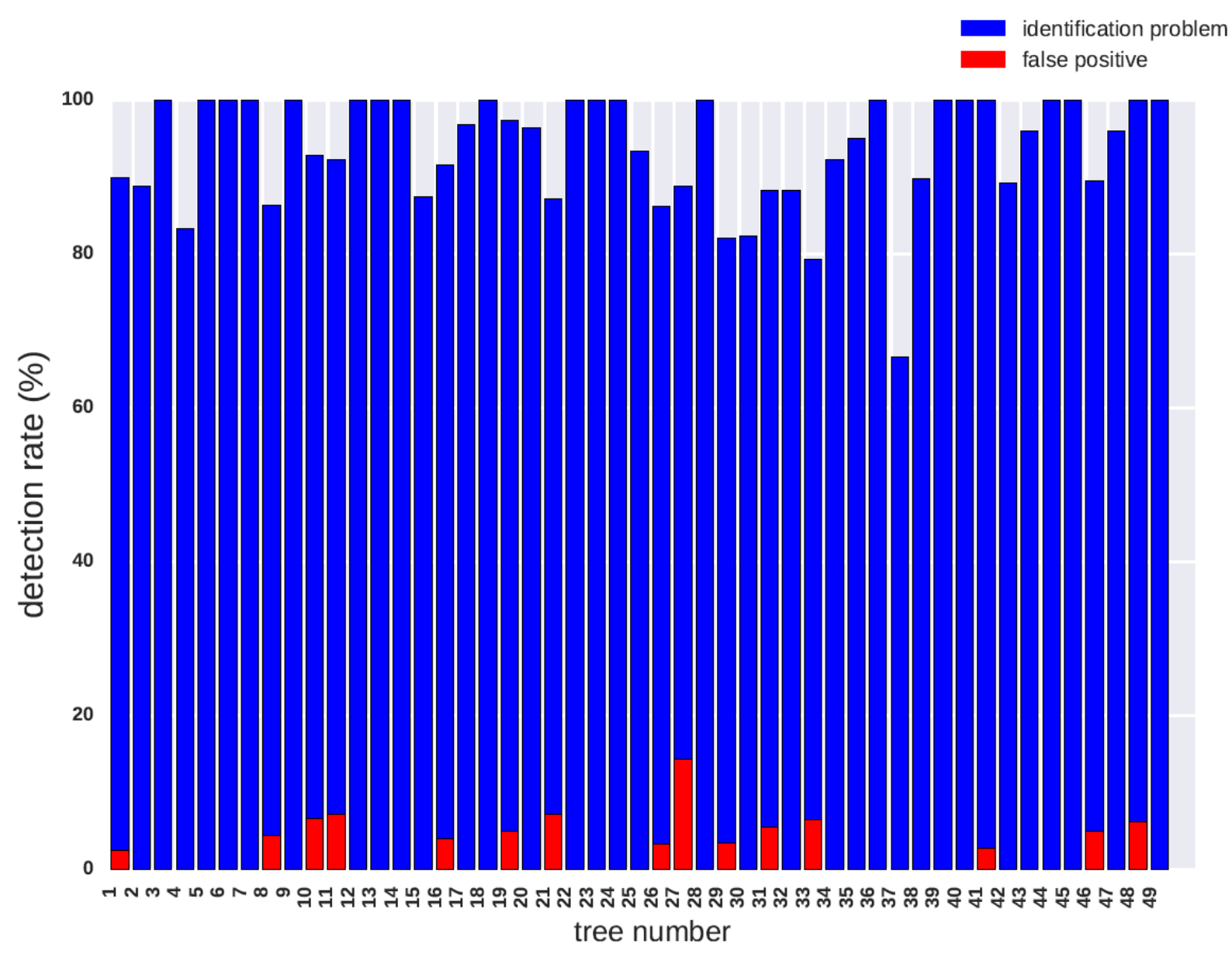

Figure 13: Plug detection rate for the noise-free case. For each of the asthmatic trees are plotted: in blue the proportion of plugged airways that are indeed predicted as plugged by the identification problem step (true positive), and in red the proportion that are predicted as plugged by the identification problem step whereas in practice they are moderately constricted at most (false positive).

Average detection rates are provided in Table 1. A more detailed analysis shows that some plugs have little influence on the ventilation distribution: they will logically not be detected by the process. This can occur because they are in regions where some other plugs have more impact or because they affect downstream generations where low flows result in low pressure drops even in cases where resistances are increased (see Figure 17). When analyzing the detection rate, one shall consider that if the tree contains only a few plugs, one missed airway notably depreciates the classification rate. In tree 37 for example, 4 plugs over 6 are actually properly classified.

Note that the space-filling tree is a model. Specifying which precise airway is plugged would require a perfect knowledge of the tree architecture even in downstream generations, which is not achievable. More reasonably, we can deduce from the analysis insights on which lung regions and tree generations are affected by plugs. This is useful information when predicting particle deposition [6] or response to Heliox treatments [5]. In Figure 14, we plot the true positive detection rate as a function of the false positive detection rate in two cases: assuming a false positive is actually properly classified if its mother or daughters airways are plugged, and the strict case from Figure 13. As summarized in Table 1 and Figure 16, the detection rate is slightly improved by $0.5 \%$ while the false positive rate is reduced by $29 \%$. 


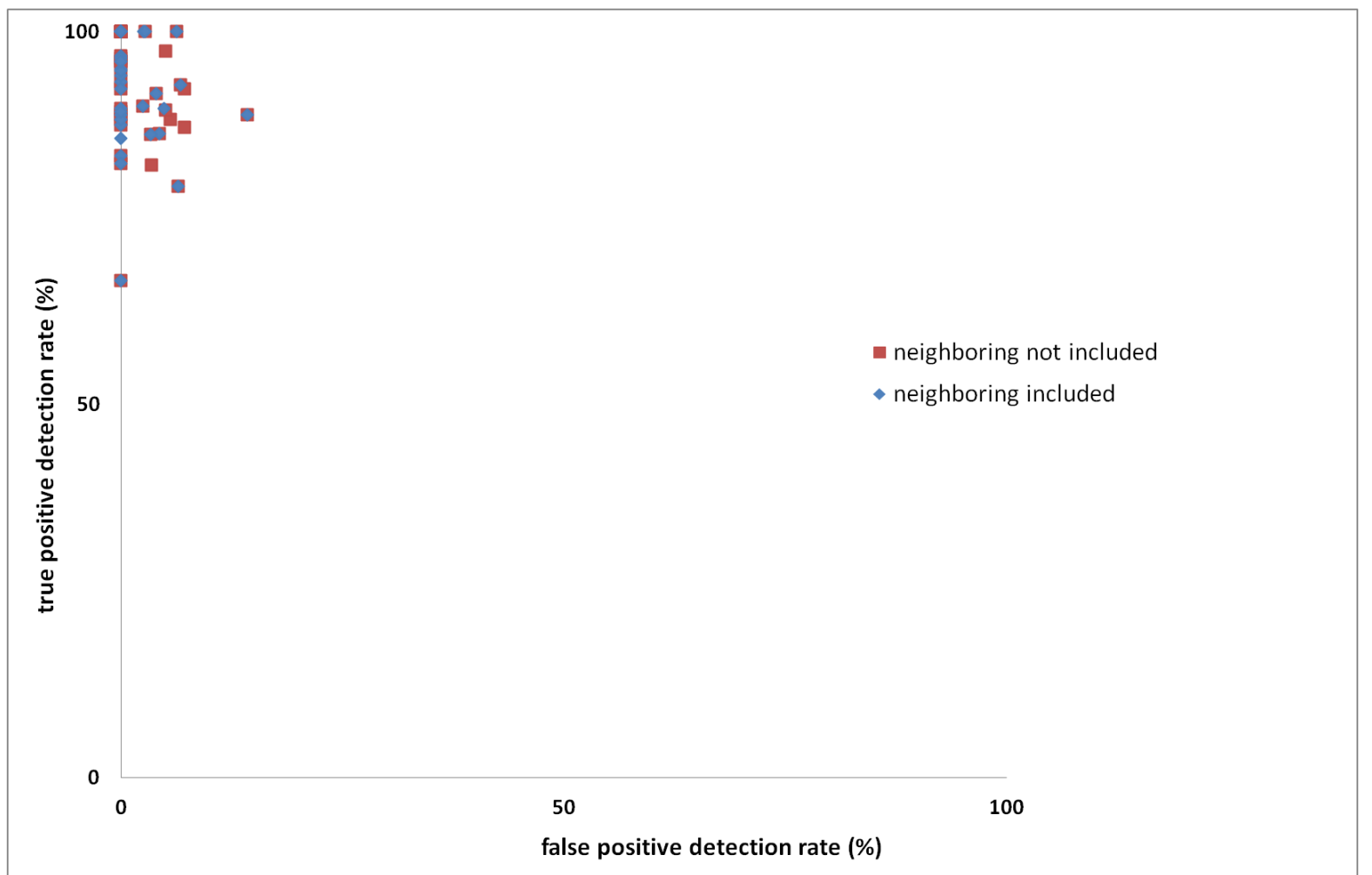

Figure 14: The true positive detection rate as a function of false positive detection rate plotted for each of the asthmatic trees. In blue, results obtained when including airway neighbors in the analysis: it is considered that if a moderately constricted airway is predicted as plugged by the identification problem step and has a mother or daughter that is actually plugged, this airway is a true positive rather than a false positive. In blue, results obtained when the neighbors are not included in the analysis. In both cases, the identification problem is performed on noise-free data.

In the following, we perform a similar analysis in the cases where the velocity field used as input of the identification problem step is noisy (see subsection 3.1.2). As shown in Figure 15, as expected, detection rates are reduced compared to the case without noise. However a large majority (86.5\%) of the plugs are detected and the rate of false positive remains low (see Table 1). 


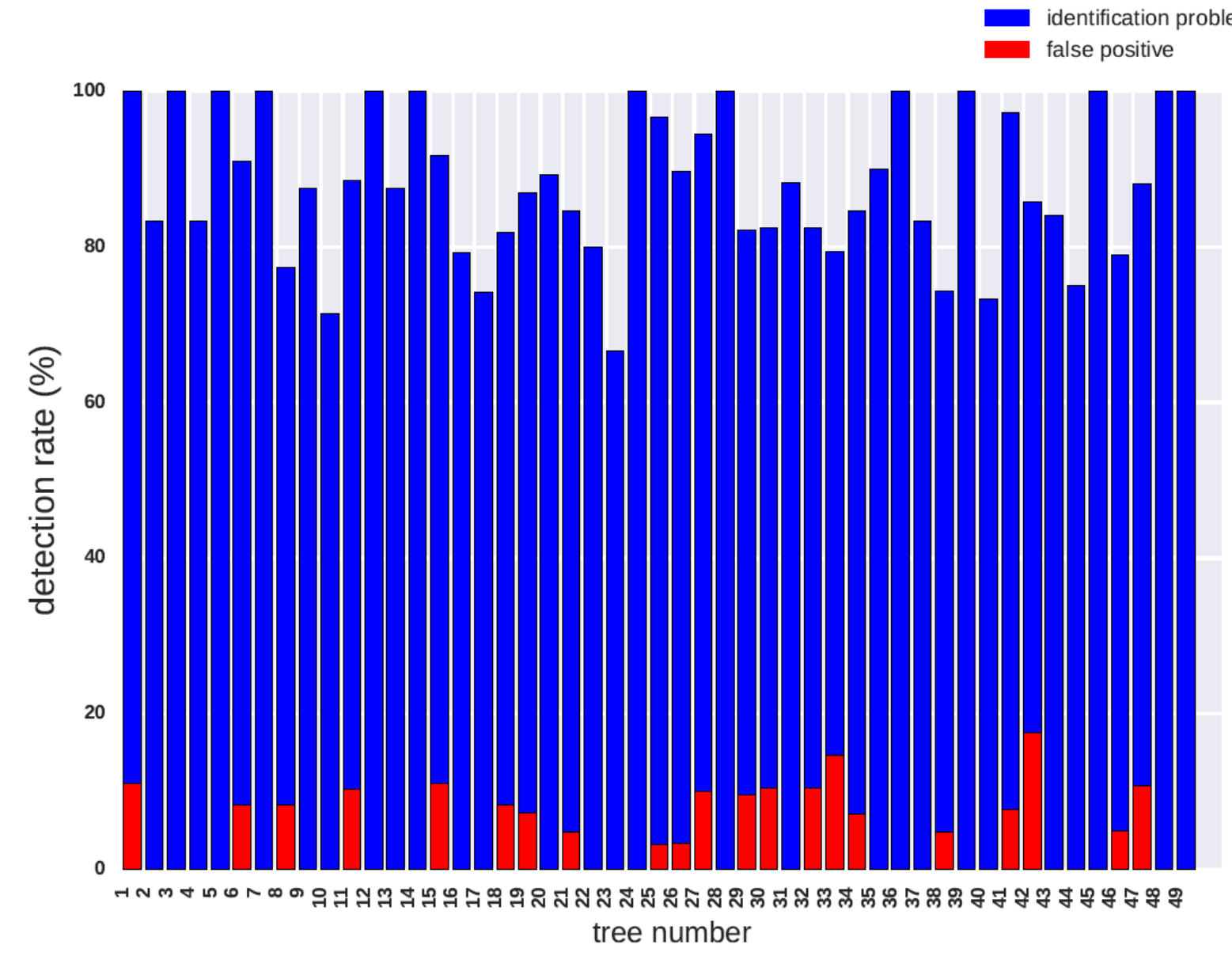

Figure 15: Plug detection rate for noisy displacement data. For each of the asthmatic trees, plotted in blue is the proportion of plugged airways that are indeed predicted as plugged after the identification problem step, in red the proportion that are predicted as plugged by the identification problem step whereas in practice they are moderately constricted at most; these are false positive. If a moderately constricted airway is predicted as plugged and it has a mother or daughter that is actually plugged, classification is judged to be good and this airway is not considered as a false positive. 
In Figure 16, detection rates obtained in the noise-free and noisy cases are compared.

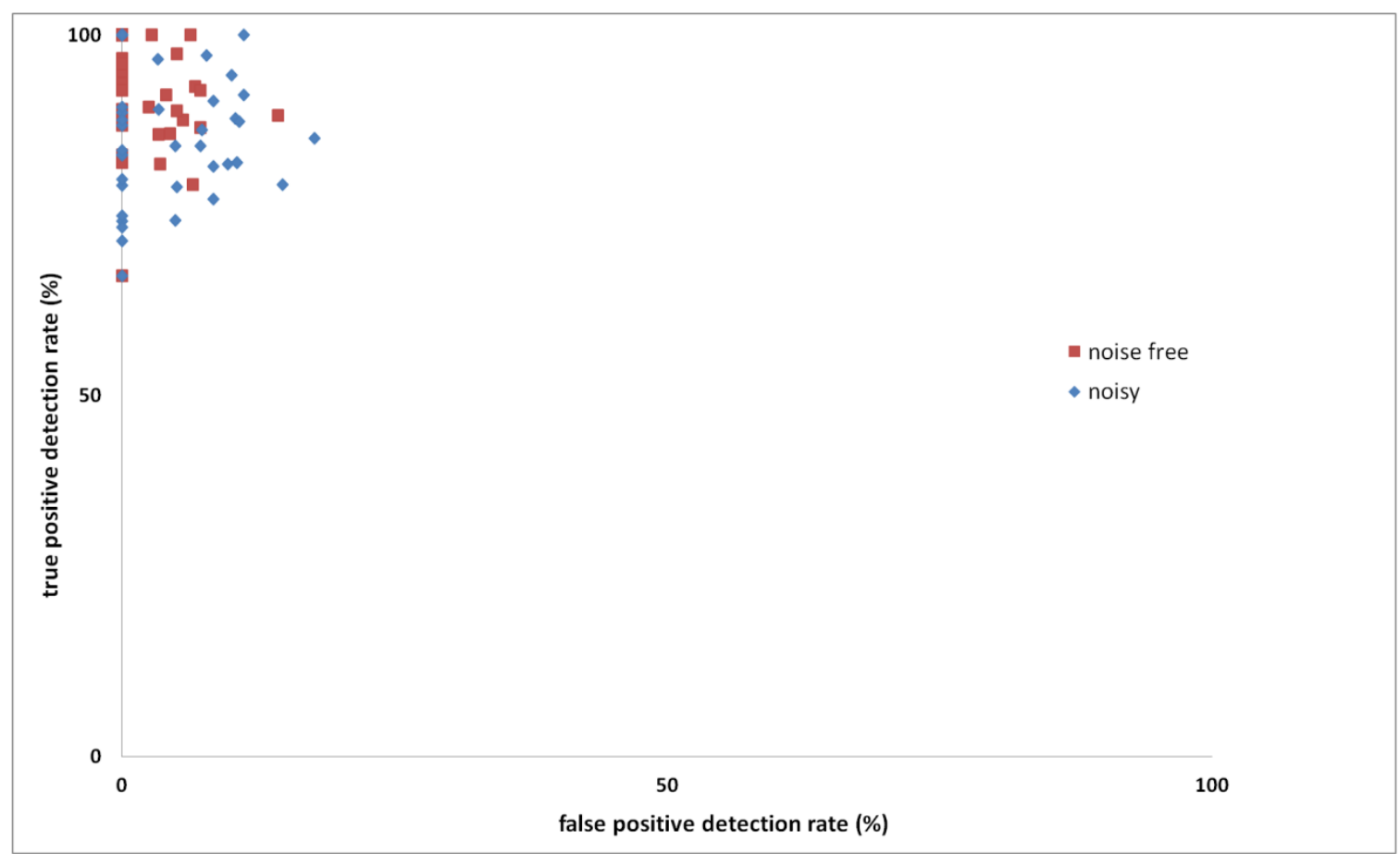

Figure 16: The true positive detection rate as a function of false positive detection rate plotted for each of the asthmatic tree. In red, results obtained when the inverse problem step is performed on noise-free data, in blue results obtained when it is performed on noisy data.

In the following, we analyze the detection rate by generation. Plugs in upper airways have a high influence on ventilation distribution [5] in contrast to constrictions that occur in distal generations. It is thus expected that dynamical ventilation data enables access to constriction information in proximal tree regions, which are critical, rather than in downstream areas which have less impact. In Figure 17 are plotted the identification problem detection rates per generation. Note that we work on a half lung, fed by an airway belonging to generation one. In simulated cases, there is no constriction on this airway since there needs to be an unknown-free path for the system to be invertible (see subsection 2.3). In practice, bronchoscopy can be used to determine if plugs exist in the most proximal airways [41], which can thus be removed from the unknown set. 


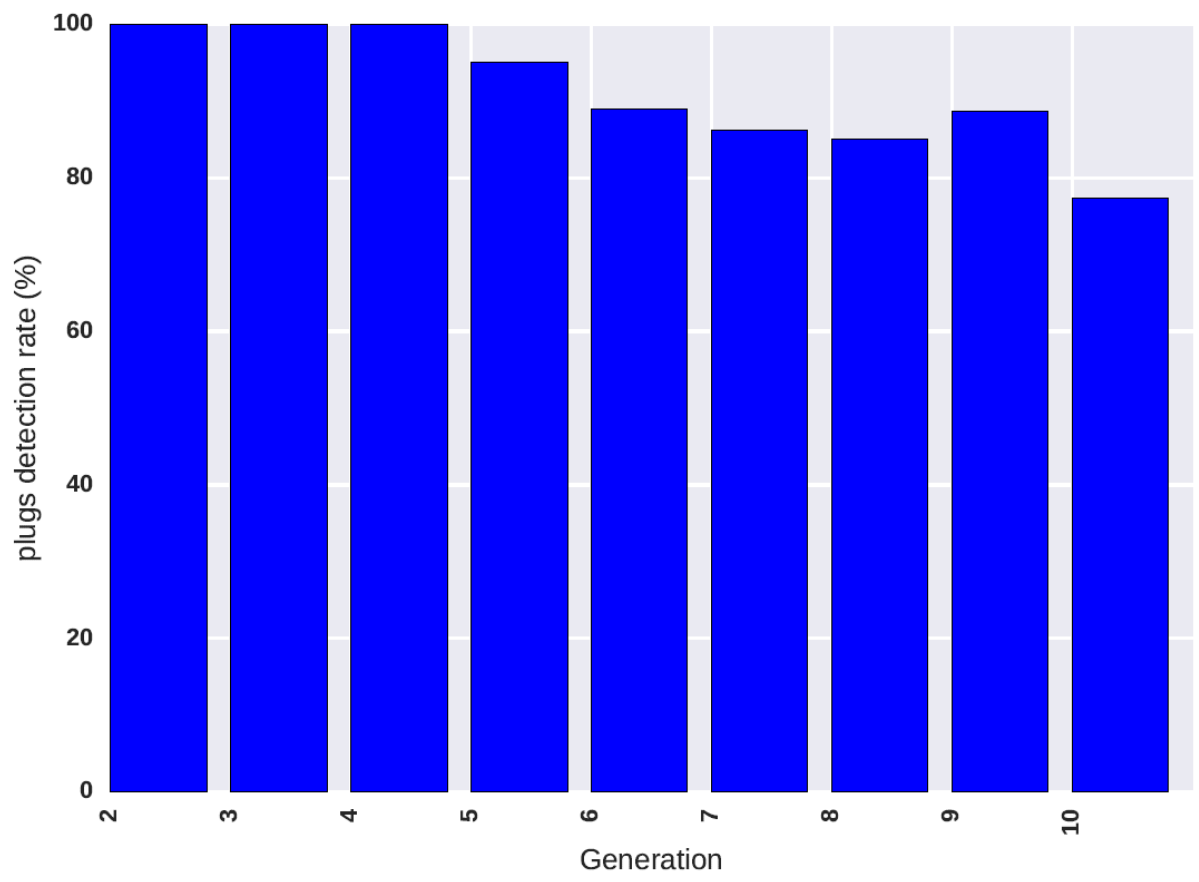

Figure 17: plugs detection rate per generation, for noisy displacement data. For each generation starting at two (one being the trachea), the identification problem plug detection rates are plotted in average over the $\mathbf{4 9}$ asthmatic trees.

Plugs in proximal generations are all detected. The detection rate drops under $80 \%$ at generation 10 . The tree has a dyadic structure, and thus there are about two times more airways at generation $n+1$ that at generation $n$. So a bad detection rate at a distal generation has a large influence on the overall detection rate result although plugs in these airways have less impact on ventilation distribution. In Table 1 detection rates are summarized. When not considering small airways (commonly defined as these with internal diameter inferior to $2 \mathrm{~mm}$ [9], starting around generation 8 [19]) more than $90 \%$ of the plugs are detected by the process.

\section{Limitations and conclusions}

Table 2 lists the key assumptions made on the model and on the data.

Assumptions and related limitations of the ventilation model used in this study are pointed out in a previous paper [15]. Note that our aim is to present a new methodology to locate plugs within the tracheobronchial tree towards the development of a diagnosis tool. Several limitations in the development are discussed below.

First we assume a linear elastic behavior of the lung parenchyma, which is likely to be valid for low tidal breathing frequency and displacement regimes. Although there is no consensus on which constitutive relation shall be considered [42], [43], [44], the actual parenchyma law is much more complex. The identification problem approach proposed in this paper works assuming mechanical properties are known, which is not the case in practice, here representative values from the literature were assumed. Note that the presented methodology would cope with any other constitutive relation for which parameters are known. Future work could include an inverse problem to determine both the plugs distribution and mechanical properties based on $4 \mathrm{D}$ data image registration. Some in-vivo techniques aim at determining the parenchyma mechanical properties based on image analysis [45], though in cases where the ventilation is strongly affected by pathological patterns within the tree, the tree-parenchyma coupling shall be taken into account. Indeed, some regions with normal mechanical behavior may not inflate because they are 
irrigated by constricted paths. To that end identification problems for parenchyma mechanical properties and plugs distribution may be coupled.

Moreover, as mentioned in subsection 3.1.3, we assume the generated space-filling tree is a proper approximation of the real one in terms of topology. Although it has good statistical properties [46], this remains a model. Validation of this topology and of the predicted constriction ratios on imaging data of high resolution is warranted. If tree bifurcations positions were not precisely known, one could consider using this methodology to develop greater global insight on which regions of the tree contain severe constrictions.

Another limitation of our work is, as stated in subsection 2.2, the use of the Pedley resistance model during expiration. In addition, airways are assumed to be rigid which is not the case in practice. Their dimensions may evolve along the breathing cycle, though this has little impact in the tidal breathing regime (see [15]). Note that a quasi-static evolution of the airway radius would be compatible with the proposed framework. Note also that, as mentioned is subsection 3.1.2, the methodology proposed in this article uses 4D ventilation data that could be noisy. The noise here accounts for registration errors, but not for gross artifacts. The latter should be further investigated. Note that some registration methods specifically designed to reduce sensibility to artifacts have been proposed, with promising results [47].

Finally, in this study, the $4 D$ ventilation target map used was built in a synthetic way adding noise to the obtained simulation result, and the process was tested based on these data. Consequently the method should ideally be tested on real experimental data for the target map (the learning base still being a synthetic one.

In conclusion, we proposed a plug detection approach that couples machine learning and ventilation modeling. Considering common 4D-CT/4D-MRI temporal and spatial resolutions we obtain very encouraging results. For noisy synthetic data more than $85 \%$ of the plugs are detected and when not considering severe constrictions in small airways, which have a lower impact on ventilation distribution, the detection rate reaches more than $90 \%$.

\section{Appendix}

\section{A.1 On the dimension of $\operatorname{ker}\left(B^{T}\right)$}

Let us compute the kernel of $B^{T}$ (see subsection 2.2 for the definition of matrix $B$ ). For a vector $P=\left(\begin{array}{c}p_{1} \\ \vdots \\ p_{N}\end{array}\right)$ we have

$$
\left(B^{T} P\right)_{j}=\left(\sum_{i} p_{i} \int_{\Omega_{i}} \operatorname{div}\left(\boldsymbol{e}_{\boldsymbol{j}}\right)\right)_{j}
$$

where $\left(\boldsymbol{e}_{\boldsymbol{j}}\right)_{j}$ are the finite element basis functions. With the imposed Dirichlet boundary conditions, they are such that $\int_{\Omega} \operatorname{div}\left(\boldsymbol{e}_{\boldsymbol{j}}\right)=0$. Let $P$ be a non-null vector such that $B^{T} P=0$. Let us consider the $k^{\text {th }}$ element of $B^{T} P$ :

$$
\left(B^{T} P\right)_{k}=\sum_{i} p_{i} \int_{\Omega_{i}} \operatorname{div}\left(\boldsymbol{e}_{\boldsymbol{k}}\right)
$$


For $e_{k}$ with support included in $\Omega_{l}$, we have that $\left(B^{T} P\right)_{k}$ is always equal to zero, indeed for $i \neq l$ $\int_{\Omega_{i}} \operatorname{div}\left(\boldsymbol{e}_{\boldsymbol{k}}\right)=\int_{\Omega} \operatorname{div}\left(\boldsymbol{e}_{\boldsymbol{k}}\right)=\int_{\partial \Omega} \boldsymbol{e}_{\boldsymbol{k}} \cdot \boldsymbol{n}=0$ since $\boldsymbol{e}_{\boldsymbol{k}}=0$ on $\partial \Omega$. And $\int_{\Omega_{l}} \operatorname{div}\left(\boldsymbol{e}_{\boldsymbol{k}}\right)=\int_{\Omega} \operatorname{div}\left(\boldsymbol{e}_{\boldsymbol{k}}\right)=0$. If $\boldsymbol{e}_{\boldsymbol{k}}$ has a support intersecting the border between regions $\Omega_{l}$ and $\Omega_{j}$ then $\int_{\Omega_{j} \cup \Omega_{l}} \operatorname{div}\left(\boldsymbol{e}_{k}\right)=0$ and

$$
\left(B^{T} P\right)_{k}=p_{l} \int_{\Omega_{l}} \operatorname{div}\left(\boldsymbol{e}_{\boldsymbol{k}}\right)+p_{j} \int_{\Omega_{j}} \operatorname{div}\left(\boldsymbol{e}_{\boldsymbol{k}}\right)=\left(p_{l}-p_{j}\right) \int_{\Omega_{l}} \operatorname{div}\left(\boldsymbol{e}_{\boldsymbol{k}}\right)
$$

So $\left(B^{T} P\right)_{k}=0 \Rightarrow p_{l}=p_{j}$. As for any two neighboring regions $\Omega_{j}$ and $\Omega_{l}$ there exists at least one $\boldsymbol{e}_{\boldsymbol{k}}$ with support included in $\Omega_{j} \cup \Omega_{l}$, we conclude that $\exists p_{0}$ such that for any $j, l \in\{1 \ldots N\}, p_{j}=p_{l}=p_{0}$. Finally we have

The reverse is trivial.

$$
B^{T} P=0 \Rightarrow \exists p_{0} \in \mathbb{R} / P=p_{0}\left(\begin{array}{c}
1 \\
\vdots \\
1
\end{array}\right) \text {. }
$$

So $\operatorname{ker}\left(B^{T}\right)=\operatorname{span}\left\{\left(\begin{array}{c}1 \\ \vdots \\ 1\end{array}\right)\right\}$ and $\operatorname{dim}\left(\operatorname{ker}\left(B^{T}\right)\right)=1$.

\section{A.2 On the reason why if $Z_{b}$ is not invertible, it has some collinear lines.}

Let $Z_{b}$ the matrix defined by (14) (see subsection 2.3). A given line $i$ of $Z_{b}$ contains 1 at position $j$ if the two following conditions are satisfied: resistance $R_{j}$ belongs to the set of unknown resistances, and the airway associated to $R_{j}$ belongs to path $T_{i}$; otherwise the line contains 0 at position $j$.

Lemma: assume $\operatorname{rank}\left(Z_{b}\right)<b$, then any line of $Z_{b}$ cannot be linear combination of several of the others and thus, necessarily, $Z_{b}$ has some collinear lines.

Proof of the lemma: we denote $\mathcal{U}$ the set of airways $\left(a_{i}\right)_{i=1 \ldots b-1}$ excluding the mother branch and which resistances are unknowns. A path $T_{l}$ goes from the mother branch down to exit $l$. Along this path, only a subset $\mathcal{A}_{l}$ of airways belongs to $U$. Let us denote $g_{l}$ the $l^{\text {th }}$ line of $Z_{b}$.

Let us note, $m_{l}=\sum_{k}\left[Z_{b}\right]_{l k}=\sum_{k} g_{l_{k}}=\operatorname{cardinal}\left(\mathcal{A}_{l}\right)$ and $\mathcal{G}=\left\{g_{l} / \forall j \neq l, m_{l} \geq m_{j}\right\}$ the set of lines containing most ones. We randomly select a line $g_{s}$ in $\mathcal{G}$. We will show that $g_{s}$ cannot be a linear combination of several vectors $g_{t \neq s}$. Let $a_{d}$ be the most distal airway of $\mathcal{A}_{s}$. Path $T_{s}$ is the only one that contains $a_{d}$. If another path $\Gamma_{q}$ contained $a_{d}$, because $a_{d}$ is the most distal branch of $T_{s}$, the tree structure would impose $\mathcal{A}_{s} \subset \mathcal{A}_{q}$. If $\mathcal{A}_{s} \neq \mathcal{A}_{q}$ then $m_{q}>m_{s}$ which is contradiction with the fact $g_{s} \in \mathcal{G}$. Thus $\mathcal{A}_{s}=\mathcal{A}_{q}$. Finally we have that $g_{s_{d}}=1$ and $\forall j \neq s, g_{j_{d}}=0$, so $g_{s}$ cannot be a linear combination of several $g_{t, \forall t \neq s}$. Repeating the same process $b-1$ times with vectors $g_{t, t \neq s}$ we conclude that any line of $Z_{b}$ cannot be linear combination of several the others.

\section{A.3 Resistance removal algorithm.}

Here we describe the algorithm used to perform the resistance removal step of the identification problem (see subsection 2.3). This step consists in reducing the linear system (15) to obtain an invertible one. Indeed, $Z_{N}$ may not be invertible. The algorithm takes advantage of the a priori prediction made on the likeliness constriction of a branch and on the lemma proved in Appendix A.2 based on the particular structure of $Z_{N}$. Remember moreover that the resistance $R_{0,0}$ is kept as an unknown in this process (see Remark 4). 
In the following, for a vector $V$ of $\mathbb{R}^{p}$ and an integer $c \leq p$, we denote by $V\{c\}$ the vector of $\mathbb{R}^{p-1}$ obtained by removing the $c^{t h}$ element of $V$. For a matrix $M$ of $\mathcal{M}_{p}(\mathbb{R})$, we denote by $M\left\{c_{1}, c_{2}\right\}$ the matrix of $\mathcal{M}_{p-1}(\mathbb{R})$ obtained by removing the $c_{1}^{\text {th }}$ line and $c_{2}^{\text {th }}$ column of $M$. For a matrix $M$ we denote $M(c,:)$ the $c^{\text {th }}$ row of $M$. Vector and matrix indexations start at one.

Figure 18 summarizes the algorithm that reduces the system to an invertible one of maximal rank by removing some well chosen column and raw. 
Initialization:

- Index exits in $\{1, \ldots, N\}$

- $l=N$

- $Z_{l}=Z_{N}$

- $k=0$

While $\operatorname{rank}\left(Z_{l}\right) \neq l$

- $\quad$ step $1 k \leftarrow k+1$

$$
\begin{gathered}
\text { If } \zeta_{l}^{-1}(k)=(0,0) \\
\circ k \leftarrow k+1
\end{gathered}
$$

- $\operatorname{step} 2 i=0, j=0, s=0$

While $s=0$

○ $i=i+1$

○ For $j^{\prime}=i+1$ to $l$

- If $Z_{l}\left(j^{\prime},:\right)$ collinear to $Z_{l}(i,:)$

- $s=1$

- $j=j^{\prime}$

- $Z_{l-1}^{j}=Z_{l}\{j, k\}$

- Exitfor

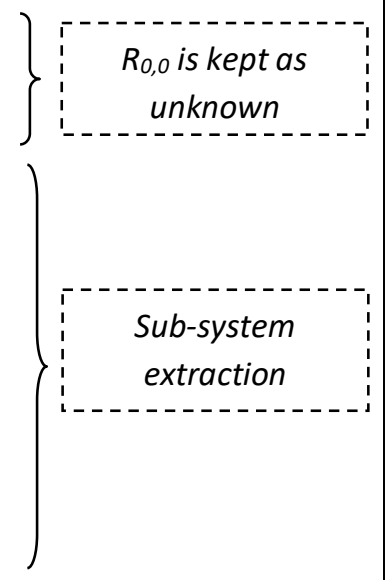

End while

If $\operatorname{rank}\left(Z_{l-1}^{j}\right)=\operatorname{rank}\left(Z_{l}\right)$

○ $\quad \operatorname{fr} \operatorname{rank}\left(Z_{l-1}^{j}\right)=l-1$

- Set $R_{k}$ as healthy

- $Z_{l-1}=Z_{l-1}^{j}$

- $G_{l-1}=G_{l}\{k\}$

- $H_{l-1}=H_{l}\{j\}$

- $l \leftarrow l-1$

$\circ$ Else

- $Z_{l-1}=Z_{l-1}^{j}$

- $U_{l-1}=U_{l}\left\{\zeta_{l}^{-1}(k)\right\}$

- Set $\zeta_{l-1}$ a function from $U_{l-1}$ to $\llbracket 1 ; l-1 \rrbracket$ based on the a priori prediction process*

- Reindex exits $\{1, \ldots, j-1\} \cup\{j+1, \ldots, l\}$ in the interval $\{1, \ldots, l-1\}$

- $l \leftarrow l-1$

Else if $\operatorname{rank}\left(Z_{l-1}^{j}\right)<\operatorname{rank}\left(Z_{l}\right)$

- Proceed to step 1

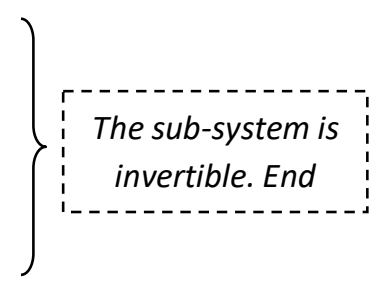

$\zeta_{l-1}$ is chosen such that unknown $R_{1}$ is the resistance associated with the airway that has highest predicted reduction ratio by the a priori prediction process, $R_{2}$ with the airway with second highest predicted reduction ratio,... and $R_{l-1}$ with the airway with lowest predicted reduction ratio.

Figure 18: Resistance removal algorithm for unknown selection. Resistances are removed one by one according to the predicted radius reduction ratio in order to make the system (15) invertible.

This algorithm converges: indeed if $l=1$ then $Z=Z_{1}=[1]$ which is invertible. 


\section{A.4 Ventilation features for the machine learning process}

In the following, we define the ventilation features used in the machine learning process (see subsection 2.4.2). Variables with subscript "asthma" refer to the ventilation in an asthmatic case while "healthy" refers to the one simulated when the tree has no bronchoconstriction.

As noted in subsections 2.4.2, we know the flow rate evolution and hence the volume of gas transiting in every airway over a breathing cycle. For the airway $(n, k)$ we note respectively $V_{n, k}^{\max }$ and $V_{n, k}^{\min }$ the maximum and minimum of $V_{n, k}(t)=\int_{0}^{t} q_{n, k}$.

We define the following feature:

$$
c_{n, k}=\frac{\left(V_{n, k}^{\max }-V_{n, k}^{\min }\right)_{\text {asthma }}-\left(V_{n, k}^{\max }-V_{n, k}^{\min }\right)_{h e a l t h y}}{\left(V_{n, k}^{\max }-V_{n, k}^{\min }\right)_{h e a l t h y}} .
$$

If $c_{n, k}<0$, less flow goes through airway $(n, k)$ than in the healthy reference case. This may indicate there are constrictions in the paths going through $(n, k)$. They could be upstream, downstream or both. The value of $c_{n, k}$ correlates with the constriction severity. To get more insight on where constrictions could occur, we define:

$$
\alpha_{n, k}=\left\{\begin{array}{cc}
1, & \text { if } c_{\left.n-1, \frac{k}{2}\right]}>0 \\
0, & \text { else. }
\end{array}\right.
$$

Note that airway $\left(n-1,\left[\frac{k}{2}\right]\right)$ is the mother of $(n, k)$. A value of 1 indicates that there is more flow going through the mother than in the healthy case. In this case the branch is not likely to be constricted and constriction may more likely be downstream. On the contrary, when the value is 0 , the mother or ascending airway may more likely be constricted. In the same spirit we define:

$$
\left\{\begin{array}{l}
\beta_{n, 2 k}= \begin{cases}1, & \text { if } c_{n, 2 k+1}>0, \\
0, & \text { else },\end{cases} \\
\beta_{n, 2 k+1}= \begin{cases}1, & \text { if } c_{n, 2 k}>0, \\
0, & \text { else. }\end{cases}
\end{array}\right.
$$

If $c_{n, k}<0$ and $\beta_{n, k}=0$, both airway $(n, k)$ and its sister have reduced flows compared to the healthy case, so some constrictions may exist upstream. To add information on the downstream environment we define:

$$
\gamma_{n, k}=\left\{\begin{array}{cc}
2, & \text { if } c_{n+1,2 k}>0 \text { and } c_{n+1,2 k+1}>0 \\
0, & \text { if } c_{n+1,2 k}<0 \text { and } c_{n+1,2 k+1}<0 \\
1, & \text { else. }
\end{array}\right.
$$

Airways $(n+1,2 k)$ and $(n+1,2 k+1)$ are the daughters of airway $(n, k)$. For a value of 0 , both daughters have reduced flows compared to the healthy case so airway $(n, k)$ or ascending branches may be constricted. If the value is 1 some constrictions would more likely occur downstream.

We also define the distribution ratio: 


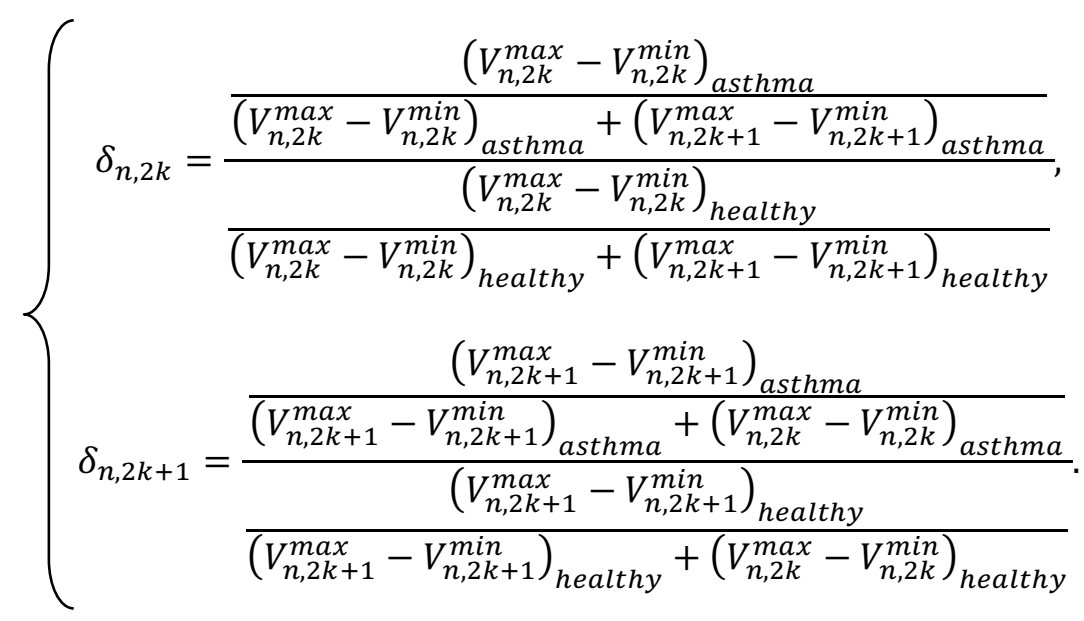

It compares the volume distribution between an airway and its sister. If $\delta_{n, k}$ is significantly smaller than 1 , airway $(n, k)$ or some downstream branches may be constricted. These features may behave differently depending on the airway generation as the effect of constrictions depends on the flow which itself decreases with the generation level [5]. So we add the generation level $n$ as a feature.

Each airway $(n, k)$ is associated with a feature vector $\left[c_{n, k}, \alpha_{n, k}, \beta_{n, k}, \gamma_{n, k}, \delta_{n, k}, n\right]$.

The machine learning step then matches the defined feature vector to the constriction ratio (12) of the considered branch. 


\section{References}

[1] C Kotaru et al., "Morphometric changes after thermal and methacholine bronchoprovocations," J Appl Physiol, no. 98(3):1028-36, 1985.

[2] M Montaudon et al., "Bronchial measurements in patients with asthma: comparison of quantitative thin-section CT findings with those in healthy subjects and correlation with pathologic findings," Radiology, vol. 253(3):844-53, 2009.

[3] D Leary et al., "Hyperpolarized He3 magnetic resonance imaging ventilation defects in asthma: relationship to airway mechanics," no. 2016 Apr;4(7), 2016.

[4] J, Parameswaran, H Lui, M Albert, and K Lutchen, "Linking ventilation heterogeneity quatified via hyperpolarized He3 MRI to dynamic lung mechanics and airway hyperresponsiveness," PLos One, no. 16;10(11):e0142738, 2015.

[5] N Pozin et al., "Calculated ventilation and effort distriubtion as a measure of respiratory disease and Heliox effectiveness," J. Biomech., vol. 60, pp. 100-109, July 2017.

[6] S Taheriann, H Rahai, BZ Gomez, T Waddington, and JR Bonifacio, "Tracheal stenosis: a CFD approach for evaluation of drug delivery.," in ASME International Mechanical Engineering Congress and Exposition, 2015.

[7] P Grenier, C Fetita, and PY Brillet, "Quantitative computed tomography imaging of airway remodelling in severe asthma," Quant Imaging Med Surg, no. 6(1):76-83, 2016.

[8] S Montesantos et al., "Airway morphology from high resolution computed tomography in healthy subjects and patients with moderate persistent asthma," The anatomical record, no. 296:852-866, 2013.

[9] PR Burgel, J de Blic, and P Chanez, "Update on the roles of distal airways in ashtma," Eur Respir Rev, no. 18:80-95, 2009.

[10] DH Carr, S Hibon, M Rubens, and KF Chung, "Peripheral airways obstruction on high-resolution computed tomography in chronic severe asthma," Respir Med, no. 92(3):448-53, 1998.

[11] N Jahani et al., "Comparison of regional ventilation and lug deformation for asthmatic vs. healthy human lungs using 4d-CT image data," J Appl Physiol, no. 15;119(10):1064-74, 2016.

[12] N Jahani et al., "A four-dimensional computed tomography comparison of healthy and asthmatic human lung," J Biomech, no. 3;56-102-110, 2017.

[13] C Kolb et al., "Regional lung ventilation analysis using temporally resolved magnetic resonance imaging," J Comput Assist Tomogr, no. 40(6):899-906, 2016. 
[14] J Biederer et al., "MRI of the lung (2/3). Why. when. how?," Insights Imaging, no. 3(4):355-71, 2012.

[15] N Pozin et al., "A tree-parenchyma coupled model for lung ventilation simulation," Int. J. Num. Method. Biomed. Eng., no. 10.1002/cnm.2873, pp. 1-30, 2017.

[16] S Sankaran, L Gady, and CA Taylor, "Fast computation of hemodynamic sensitivity to lumen segmentation uncertainty," IEEE trans med imaging, no. 34(12):2562-71, 2015.

[17] A Lungu et al., "Diagnosis of pulmonary hypertension from magnetic resonance imaging-based computational models and decision tree analysis," Plum circ, no. 6(2):181-90, 2016.

[18] T Aikawa, S Shimura, H Sasaki, M Ebina, and T Takishima, "Marked goblet cell hyperplasia with mucus accumulation in the airways of patients who died of severe acute asthma attack," Chest, no. 101(4):916-21, 1992.

[19] TT Soong, P Nicolaides, CP Yu, and SC Soong, "A statistical description of the human tracheobronchial tree geometry," Respir physiol, no. 37(2):161-72, 1979.

[20] B Maury, The Respiratory System in Equations.: Springer, 2013, vol. 7.

[21] M Ismail, A Comerford, and WA Wall, "Coupled and reduced dimensional modeling of respiratoy mechanics during spontaneous breathing," Int. J. Numer. Meth. Biomed. Engng, no. 29, pp. 1285-1305, 2013.

[22] TJ Pedley, RC Schroter, and MF Sudlow, "Energy losses and pressure drops in models of human airways," Respir Physiol, no. 9(3):371-86, 1970.

[23] MY Kang, J Hwang, and JW Lee, "Effect of geometric variations on pressure loss for a model bifurcation of the human lung airway," J.Biomech, no. 44(6):1196-9, 2011.

[24] P Cazeaux and C Grandmont, "Homogenization of a multiscale viscoelastic model with nonlocal damping, application to the human lungs," Mathematical Models and Methods in Applied Sciences, vol. 25(06):1125-1177, 2012.

[25] YK Mariappan, KJ Glaser, and RL Ehman, "Helium-3 magnetic resonance elastography of the lung," Clin anat, no. 23(5):497-511, 2016.

[26] EE Greenblatt et al., "Analysis of three-dimensional aerosol deposition in pharmacologically relevant terms: beyound black or white ROIs," J Aerosol Med Pulm Drig Deliv, no. 28(2):116-29, 2014.

[27] Meshlab - developped with the support of 3D-CoForm project - meshlab.sourceforge.net.

[28] C Geuzaine and JF Remacle, "Gmsh: a three-dimensional finite element mesh generator with built-in pre- and post-processing," Int. J. Numer. Meth. Engng, vol. 79(11):1309-1331, 2009.

[29] EE Greenblatt et al., "Analysis of three-dimensional aerosol deposition in pharmacologically relevant 
terms: beyound black or white ROIs," J Aerosol Med Pulm Drig Deliv, no. 28(2):116-29, 2014.

[30] S Durrleman et al., "Morphometry of anatomical shape complexes with dense deformations and sparse parameters," Neurolmage, vol. 101:35-49, 2014.

[31] V Boldea, GC Sharp, SB Jiang, and D Sarrut, "4D-CT lung motion estimation with deformable registration: quantification of motion nonlinearity and hysterisis," Med Phys, no. 35(3):1008-18, 2008.

[32] A Hilsmann et al., "Deformable 4DCT lung registration with vessel bifurcations," International journal of computer assisted radiology and surgery, no. 39015418, 2007.

[33] J Vandemeulebroucke et al., "Automated segmentation of a motion mask to preserve sliding motion in deformable registration of thoracic CT," Med Phys, no. 39(2):1006-15, 2012.

[34] J Cai, Z Chang, and Z Wang, "Four-dimensional magnetic resonance imaging (4D-MRI) using imagebased respiratory surrogate: A feasibility study," Med Phys, no. 38(12):6384-94, 2011.

[35] Y Tokihira, U Langner, BW Loo, J Shen, and PJ Keall, "Retrospective analysis of artifacts in fourdimensional CT images of 50 abdominal and thoracic radiotherapy patients," Int J Radiat Oncol Biol Phys, no. 72(4):1250-1258, 2008.

[36] S Montesantos, I Katz, M Pichelin, and G Caillibote, "The creation and statistical evaluation of a deterministic model of the human bronchial tree from HRCT images," PLOS one, 2016.

[37] MA Epstein and JR Ligas, Respiratory biomechanics, Springer-Verlag, Ed., 1990.

[38] F Pedregosa et al., "Scikit-learn: machine learning in python," JMLR, no. 12(2011):2825-2830, 2011.

[39] F Laurent, V Latrabe, C Raherison, R Marthan, and JM Tunon-de-Lara, "Functional significance of air trapping detected in moderate asthma," Eur Radiol, no. 10(9):1404-10, 2000.

[40] EE Greenblatt, JP Butler, JG Venegas, and T Winkler, "Pendelluft in the bronchial tree," J Appl Physiol, no. 117(9):979-88, 2014.

[41] H Hoppe et al., "Grading airway stenosis down to the segmental level using virtual bronchoscopy," Chest, no. 125(2):704-11, 2004.

[42] L Berger et al., "A poroelastic model coupled to a fluid network with applications in lung modelling," Int J Numer Method Biomed Eng, vol. 32(1):e02731, 2015.

[43] L Yoshihara, CJ Roth, and WA Wall, "Fluid-structure interaction including volumetric coupling with homogenised subdomains for modeling respiratory mechanics," Int J Numer Method Biomed Eng, no. 10.1002/cnm.2812, 2016.

[44] B Suki, D Stamenovic, and R Hubmayr, "Lung Parenchymal Mechanics," Compr Physiol, vol. 1(3):13171351, 2011. 
[45] AD Freed and DR Einstein, "An implicit elastic theory for lung parenchyma," Int J Eg Sci, no. 2013 Hen;62:31-47, 2013.

[46] MH Tawhai et al., "CT-based geometry analysis and finite element models of the human and ovine bronchial tree," J Appl Physiol, no. 97(6):2310-21, 2004.

[47] J Vandemeulebroucke, S Rit, J Kybuc, P Clarysse, and D Sarrut, "Spatiotemporal motion estimation for respiratory-correlated imaging of the lungs," Medical Physics, no. 10.1002/mp.12780, 2018. 


\section{Tables}

\begin{tabular}{|c|c|c|c|c|c|c|}
\hline \multirow{2}{*}{} & \multicolumn{3}{|c|}{ Noise-free } & \multicolumn{3}{c|}{ With noise } \\
\cline { 2 - 7 } & $\mathrm{ML}$ & $\mathrm{IP}$ & $\mathrm{FP}$ & $\mathrm{ML}$ & $\mathrm{IP}$ & $\mathrm{FP}$ \\
\hline No neighbors & 98.5 & 93.8 & 1.7 & 98.5 & 85.2 & 6.0 \\
\hline Neighbors & 98.5 & 94.3 & 1.2 & 98.5 & 88.0 & 3.8 \\
\hline
\end{tabular}

Table 1: Summary of plug classification and detection results, in \%, by the machine learning step (ML), by the identification problem step (IP), and proportion of false positive (FP). Note that at the ML step, a plug is considered to be properly classified if its predicted radius reduction ratio ranks in the first half (see subsection 3.2) whereas a plug is considered to be properly classified by the IP step if its average computed radius reduction ratio over the ten time points is under 0.3 (see subsection 3.2). Results are given for both noise-free and noisy displacement data.

\begin{tabular}{|l|l|}
\hline \multirow{4}{*}{ Assumptions on the model } & $\begin{array}{l}\text { - Linear elastic parenchyma behavior } \\
\text { - Homogeneous isotropic parenchyma model }\end{array}$ \\
& $\begin{array}{l}\text { - Use of Pedley resistance model both at } \\
\text { inspiration and expiration }\end{array}$ \\
\hline Assumptions on the data & $\begin{array}{l}\text { - } \text { Kigid airways } \\
\text { properties }\end{array}$ \\
& $\begin{array}{l}\text { - Knowledge of the tree bifurcations } \\
\text { positions }\end{array}$ \\
& $\begin{array}{l}\text { Radii of airways determined as not } \\
\text { constricted are assumed to be known, their } \\
\text { values being set by the space-filling tree } \\
\text { model }\end{array}$ \\
& $\begin{array}{l}4 D \text { ventilation data are known and do not } \\
\text { contain artifacts }\end{array}$ \\
\hline
\end{tabular}

Table 2: assumptions on the model and on the data. 